\title{
Mapping deuterated methanol toward L1544
}

\section{Deuterium fraction and comparison with modeling ${ }^{\star}$}

\author{
A. Chacón-Tanarro ${ }^{1}$, P. Casellii ${ }^{1}$, L. Bizzocchi ${ }^{1}$, J. E. Pineda ${ }^{1}$, O. Sipilä ${ }^{1}$, A. Vasyunin ${ }^{1,2}$, \\ S. Spezzano ${ }^{1}$, A. Punanova ${ }^{2,1}$, B. M. Giuliano ${ }^{1}$, and V. Lattanzi ${ }^{1}$

\footnotetext{
${ }^{1}$ Max-Planck-Institüt für extraterrestrische Physik, Giessenbachstrasse 1, 85748 Garching, Germany

e-mail: a.chacon. tanarro@gmail.com

${ }^{2}$ Ural Federal University, 620002, 19 Mira street, Yekaterinburg, Russia
}

Received 25 January 2018 / Accepted 17 August 2018

\begin{abstract}
Context. The study of deuteration in pre-stellar cores is important in order to understand the initial physical and chemical conditions in the process of star formation. In particular, observations toward pre-stellar cores of methanol and deuterated methanol, solely formed on the surface of dust grains, may provide useful insights into surface processes at low temperatures.

Aims. Here we analyze maps of $\mathrm{CO}$, methanol, formaldehyde, and their deuterated isotopologues toward a well-known pre-stellar core. This study allows us to test current gas-dust chemical models.

Methods. Single-dish observations of $\mathrm{CH}_{3} \mathrm{OH}, \mathrm{CH}_{2} \mathrm{DOH}, \mathrm{H}_{2} \mathrm{CO}, \mathrm{H}_{2}^{13} \mathrm{CO}, \mathrm{HDCO}, \mathrm{D}_{2} \mathrm{CO}$, and $\mathrm{C}^{17} \mathrm{O}$ toward the prototypical pre-stellar core L1544 were performed at the IRAM $30 \mathrm{~m}$ telescope. We analyze their column densities and distributions, and compare these observations with gas-grain chemical models.

Results. The maximum deuterium fraction derived for methanol is $\left[\mathrm{CH}_{2} \mathrm{DOH}\right] /\left[\mathrm{CH}_{3} \mathrm{OH}\right] \sim 0.08 \pm 0.02$, while the measured deuterium fractions of formaldehyde at the dust peak are $[\mathrm{HDCO}] /\left[\mathrm{H}_{2} \mathrm{CO}\right] \sim 0.03 \pm 0.02,\left[\mathrm{D}_{2} \mathrm{CO}\right] /\left[\mathrm{H}_{2} \mathrm{CO}\right] \sim 0.04 \pm 0.03$, and $\left[\mathrm{D}_{2} \mathrm{CO}\right] /[\mathrm{HDCO}] \sim$ $1.2 \pm 0.3$. Observations differ significantly from the predictions of models, finding discrepancies between a factor of 10 and a factor of 100 in most cases. It is clear though that to efficiently produce methanol on the surface of dust grains, quantum tunneling diffusion of $\mathrm{H}$ atoms must be switched on. It also appears that the currently adopted reactive desorption efficiency of methanol is overestimated and/or that abstraction reactions play an important role. More laboratory work is needed to shed light on the chemistry of methanol, an important precursor of complex organic molecules in space.
\end{abstract}

Key words. astrochemistry - ISM: clouds - ISM: individual objects: L1544 - stars: formation - ISM: molecules

\section{Introduction}

Pre-stellar cores form in molecular clouds, due to the influence of gravity, magnetic fields, and turbulence. They are dense $\left(n_{\mathrm{H}_{2}}>10^{5} \mathrm{~cm}^{-3}\right)$ and cold $(T<10 \mathrm{~K})$ toward their center (Keto \& Caselli 2010). They are the starting point in the process of star formation (Bergin \& Tafalla 2007; Caselli \& Ceccarelli 2012) as they are self-gravitating dense cores which present signs of contraction motions and chemical evolution (Crapsi et al. 2005). Hence, they are ideal laboratories for the study of the early evolutionary stages of low-mass star formation.

Methanol $\left(\mathrm{CH}_{3} \mathrm{OH}\right)$ is one of the most widespread organic molecules in the ISM and is a major precursor of chemical complexity in space. Methanol is believed to form on dust grain surfaces by sequential addition of hydrogen atoms to adsorbed CO molecules (Tielens \& Hagen 1982; Watanabe \& Kouchi 2002). This process is thought to take place in cold dense cores at densities above $10^{4} \mathrm{~cm}^{-3}$ where large amounts of gas-phase $\mathrm{CO}$ molecules start to freeze-out onto dust grain surfaces (Willacy et al. 1998; Caselli et al. 1999; Tafalla et al. 2002).

In L1544, Vastel et al. (2014) deduced that the $\mathrm{CH}_{3} \mathrm{OH}$ emission should trace an external layer of the core. Bizzocchi et al. (2014) mapped the $\mathrm{CH}_{3} \mathrm{OH}$ emission across

\footnotetext{
* Based on observations carried out with the IRAM 30 m Telescope. IRAM is supported by INSU/CNRS (France), MPG (Germany), and IGN (Spain)
}

L1544, finding an asymmetric ring-like shape surrounding the dust peak. The methanol peak is about 4000 au from the core center and its distribution can be reproduced by the recent gas-grain chemical model (applied to L1544) by Vasyunin et al. (2017). This can be understood by the fact that the $\mathrm{CH}_{3} \mathrm{OH}$ molecule can more easily desorb from the grain surface when a significant fraction of surface ice is in $\mathrm{CO}$, and $\mathrm{CO}$-rich dust surfaces are present around 4000-5000 au from the core center because of the fast $\mathrm{CO}$ freeze-out at that location and the different $\mathrm{CO}$ and $\mathrm{H}_{2} \mathrm{O}$ photodesorption rates (Vasyunin et al. 2017).

Deuterated methanol should follow the methanol distribution as they are both formed on the surface of dust grains. However, as already found by previous authors (Caselli et al. 2002b), the deuterium fraction increases toward the densest and coldest parts of the core where $\mathrm{CO}$ is mainly in solid form. Toward the center, due to the dissociative recombination of the abundant deuterated forms of $\mathrm{H}_{3}^{+}$, the $\mathrm{D} / \mathrm{H}$ atomic ratio increases from the cosmic value $\left(\sim 1.5 \times 10^{-5}\right.$; Linsky et al. 2006$)$ to values higher than 0.1 (e.g., Roberts et al. 2003). Thus, toward the core center, $\mathrm{D}$ atoms compete with $\mathrm{H}$ atoms in the saturation of $\mathrm{CO}$ molecules, efficiently producing deuterated methanol (Aikawa et al. 2012; Taquet et al. 2012; Ceccarelli et al. 2014). Observations toward L1544 showed that the centroid velocity $\left(V_{\mathrm{LSR}}\right)$ of $\mathrm{CH}_{2} \mathrm{DOH}$ is about $0.2 \mathrm{~km} \mathrm{~s}^{-1}$ lower than that of $\mathrm{CH}_{3} \mathrm{OH}$ (Bizzocchi et al. 2014); this shift is the same as that found when 
Table 1. Observed lines.

\begin{tabular}{ccccccc}
\hline \hline Line & $\begin{array}{c}\text { Rest frequency } \\
(\mathrm{MHz})\end{array}$ & $\begin{array}{c}\mathrm{rms} \\
(\mathrm{mK})\end{array}$ & $\begin{array}{c}\text { Velocity resolution } \\
\left(\mathrm{km} \mathrm{s}^{-1}\right)\end{array}$ & $\begin{array}{c}\text { Pixel size } \\
(\operatorname{arcsec})\end{array}$ & $F_{\text {eff }} / B_{\text {eff }}$ & References \\
\hline $\mathrm{CH}_{3} \mathrm{OH}\left(2_{0,2}-1_{0,1}, \mathrm{~A}^{+}\right)$ & $96741.375 \pm 0.003$ & 62 & 0.06 & 5 & 1.18 & 1 \\
$\mathrm{CH}_{3} \mathrm{OH}\left(2_{1,2}-1_{1,1}, \mathrm{E}_{2}\right)$ & $96739.362 \pm 0.003$ & 62 & 0.06 & 5 & 1.18 & 1 \\
$\mathrm{CH}_{3} \mathrm{OH}\left(2_{0,2}-1_{0,1}, \mathrm{E}_{1}\right)$ & $96744.550 \pm 0.003$ & 62 & 0.06 & 5 & 1.18 & 1 \\
$\mathrm{CH}_{2} \mathrm{DOH}\left(2_{0,2}-1_{0,1}, \mathrm{e}_{0}\right)$ & $89407.817 \pm 0.002$ & 15 & 0.07 & 5 & 1.18 & 2 \\
$\mathrm{CH}_{2} \mathrm{DOH}\left(3_{0,3}-2_{0,2}, \mathrm{e}_{0}\right)$ & $134065.381 \pm 0.002$ & 15 & 0.04 & 5 & 1.25 & 2 \\
$\mathrm{H}_{2} \mathrm{CO}\left(2_{1,2}-1_{1,1}\right)$ & $140839.502 \pm 0.010$ & 40 & 0.10 & 5 & 1.26 & 3,4 \\
$\mathrm{H}_{2}^{13} \mathrm{CO}\left(2_{1,2}-1_{1,1}\right)$ & $137449.950 \pm 0.004$ & 30 & 0.11 & 5 & 1.26 & 5 \\
$\mathrm{HDCO}_{2}\left(2_{1,1}-1_{1,0}\right)$ & $134284.830 \pm 0.100$ & 10 & 0.11 & 5 & 1.20 & 6 \\
$\mathrm{D}_{2} \mathrm{CO}\left(2_{1,2}-1_{1,1}\right)$ & $110837.830 \pm 0.100$ & 17 & 0.13 & 5 & 1.25 & 6 \\
$\mathrm{C}{ }^{17} \mathrm{O}(1-0)$ & $112360.007 \pm 0.015$ & 75 & 0.05 & 4 & 1.20 & 7 \\
\hline
\end{tabular}

Notes. Columns are: rest frequency, noise level, velocity resolution, map pixel size, forward and beam efficiency ratios, and the references for the spectroscopic information for each line.

References. (1) Xu \& Lovas (1997); (2) Pearson et al. (2012); (3) Müller \& Lewen (2017); (4) Cornet \& Winnewisser (1980); (5) Muller et al. (2000); (6) Bocquet et al. (1999); (7) Klapper et al. (2003). The references and values from (3) to (7) were found using the CDMS (Cologne Database for Molecular Spectroscopy; Müller et al. 2001, 2005).

comparing the $V_{\mathrm{LSR}}$ of $\mathrm{C}^{17} \mathrm{O}(1-0)$, which traces the outer parts of the core, with the $V_{\mathrm{LSR}}$ of $\mathrm{N}_{2} \mathrm{D}^{+}(2-1)$, mainly tracing the dense central regions where $\mathrm{CO}$ is frozen out (Caselli et al. 2002a), thus suggesting that $\mathrm{CH}_{2} \mathrm{DOH}$ is tracing denser regions than $\mathrm{CH}_{3} \mathrm{OH}$, in agreement with the theoretical expectations. However, this hypothesis is based on the results of single pointing observations, so no information on the spatial distribution of $\mathrm{CH}_{2} \mathrm{DOH}$ in the core can be inferred.

Formaldehyde $\left(\mathrm{H}_{2} \mathrm{CO}\right)$ can be formed both via grain-surface chemistry (in an intermediate step of the formation of methanol) and gas-phase chemistry (e.g., through reactions of hydrocarbons with oxygen atoms; Yamamoto 2017). Which route actually dominates is still a matter of debate (Parise et al. 2006). Parise et al. (2009) suggested that pure gas-phase chemistry can account for the abundances of formaldehyde and its deuterated species observed toward the Orion Bar PDR. On the other hand, Bergman et al. (2011) had to invoke grain-surface chemistry to explain the high deuteration found in the $\rho$ Oph A cloud. Interestingly, these authors found $\mathrm{D}_{2} \mathrm{CO}$ to be more abundant than HDCO.

The deuterium fraction measured in $\mathrm{N}_{2} \mathrm{H}^{+}, \mathrm{NH}_{3}, \mathrm{H}_{2} \mathrm{CO}$, and $\mathrm{CH}_{3} \mathrm{OH}$ (Emprechtinger et al. 2009; Bianchi et al. 2017a) reaches the highest values in dynamically evolved starless cores (prestellar cores) and toward the youngest protostellar objects, while it decreases in more evolved phases in the low-mass star formation process. The study of different evolutionary stages is thus important to be able to understand how the deuterium budget builds up in molecules formed preferentially in the gas phase and on dust grain surfaces, and to follow these processes during the dynamical evolution of star forming clouds. We focus our investigation on L1544, a nearby well-known pre-stellar core in the Taurus Molecular Cloud. Here, we study the spatial features of the methanol and formaldehyde deuteration, together with the $\mathrm{CO}$ distribution as $\mathrm{CO}$ is thought to be the parent molecule of methanol. The objective is to gain insights into the surface chemistry and the early history of deuteration during the formation of low-mass stars, which is still unknown.

The structure of the paper is as follows. In Sect. 2 we describe the observations, followed by the results in Sect. 3. The analysis, comparison with models, and discussion are presented in Sects. 4-6, respectively. Our findings are summarized in Sect. 7.

\section{Observations}

The data presented here were observed using the IRAM $30 \mathrm{~m}$ telescope, located at Pico Veleta (Spain), during four observing sessions in 2014 and 2015. We obtained on-the-fly (OTF) maps for 3 and $2 \mathrm{~mm}$ emission lines of the molecules: $\mathrm{CH}_{2} \mathrm{DOH}$, $\mathrm{CH}_{3} \mathrm{OH}, \mathrm{H}_{2} \mathrm{CO}, \mathrm{H}_{2}^{13} \mathrm{CO}, \mathrm{HDCO}, \mathrm{D}_{2} \mathrm{CO}$, and $\mathrm{C}^{17} \mathrm{O}$ (see Table 1). A region of $2.5^{\prime} \times 2.5^{\prime}$ was mapped in all the lines, except $\mathrm{C}^{17} \mathrm{O}$, for which a $4^{\prime} \times 4^{\prime}$ OTF map was performed. We applied beam switching for these maps. The pointing accuracy is $<4^{\prime \prime}$. Various E090/E150 Eight MIxer Receiver (EMIR) heterodyne receiver setups were adopted, and the VErsatile SPectrometer Assembly (VESPA) was used as backend for $\mathrm{CH}_{3} \mathrm{OH}, \mathrm{CH}_{2} \mathrm{DOH}$, and $\mathrm{C}^{17} \mathrm{O}$, with a spectral resolution of $20 \mathrm{kHz}$. The data for formaldehyde and its deuterated species were collected using the FTS backend with a spectral resolution of $50 \mathrm{kHz}$. All the lines of $\mathrm{CH}_{3} \mathrm{OH}$ and $\mathrm{H}_{2} \mathrm{CO}$ were observed simultaneously; $\mathrm{CH}_{2} \mathrm{DOH}$ $\left(2_{0,2}-1_{0,1}\right), \mathrm{CH}_{2} \mathrm{DOH}\left(3_{0,3}-2_{0,2}\right), \mathrm{C}^{17} \mathrm{O}(1-0), \operatorname{HDCO}\left(2_{1,1}-1_{1,0}\right)$, and $\mathrm{D}_{2} \mathrm{CO}\left(2_{1,2}-1_{1,1}\right)$ were observed in other different setups. All the maps were finally convolved to a common angular resolution of $30^{\prime \prime}$, in order to facilitate the comparison between different molecules. The initial data reduction was done with GILDAS $^{1}$.

The observations were done under average good weather conditions $\left(\tau_{225 \mathrm{GHz}} \sim 0.1-0.5\right.$, i.e., pwv $\left.\sim 2-8 \mathrm{~mm}\right)$, and with $T_{\text {sys }}$ ranging from $\sim 100$ to $\sim 150 \mathrm{~K}$ for the $3 \mathrm{~mm}$ band, and from $\sim 100$ to $\sim 160 \mathrm{~K}$ for the $2 \mathrm{~mm}$ band. All spectra were converted from $T_{A}^{*}$ to $T_{\mathrm{mb}}$ using the forward and main beam efficiency ratios shown in Table 1 , due to the extended but not uniform nature of the maps presented here. We note that this may overestimate our results by $\sim 20 \%$ for the more extended emission of $\mathrm{C}^{17} \mathrm{O}(1-0)$, but this is within the uncertainties and it does not change the conclusions derived from the morphology of the emission distribution of $\mathrm{C}^{17} \mathrm{O}(1-0)$ across the map (see Sect. 3). The spectra obtained averaging the central $30 \times 30 \operatorname{arcsec}^{2}$ of each map are shown in Fig. 1.

\section{Results}

The integrated intensities were derived calculating the zeroth moment of the maps using a custom code written in Python,

1 https://www.iram.fr/IRAMFR/GILDAS/ 

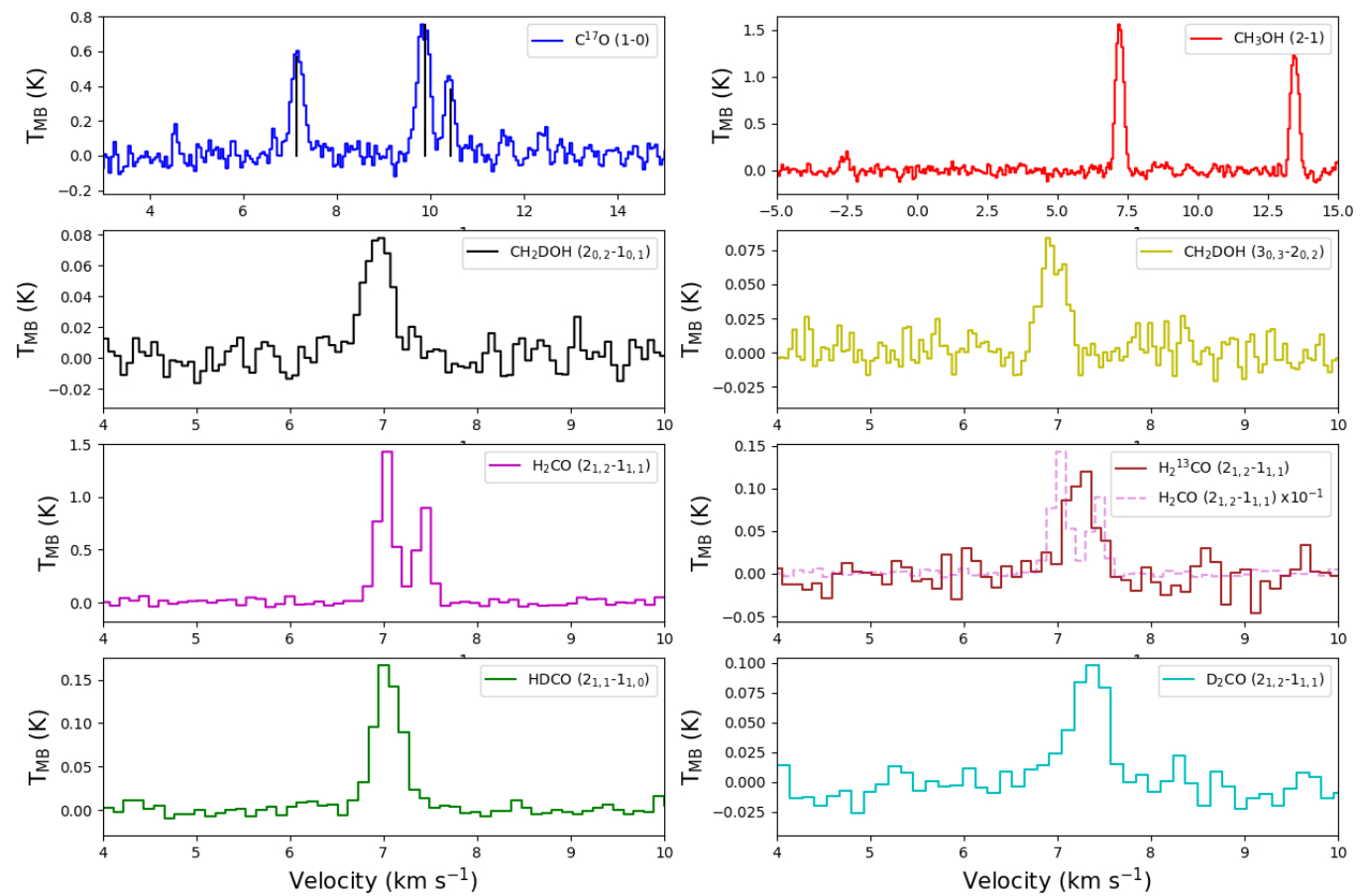

Fig. 1. Averaged spectra of all lines in the central $\sim 30 \times 30 \operatorname{arcsec}^{2}$. The specifications of the lines and the spectra are shown in Table 1 . The $\mathrm{H}_{2} \mathrm{CO}$ $\left(2_{1,2}-1_{1,1}\right)$ spectrum is superposed on the $\mathrm{H}_{2}^{13} \mathrm{CO}\left(2_{1,2}-1_{1,1}\right)$ spectrum to show that the $\mathrm{H}_{2} \mathrm{CO}\left(2_{1,2}-1_{1,1}\right)$ line is self-absorbed. Superposed on the $\mathrm{C}^{17} \mathrm{O}$ spectrum there are three lines that indicate the relative intensities of the hyperfine components.

making use of Astropy (Astropy Collaboration 2013), SciPy, and NumPy (van der Walt et al. 2011). In this section only some of the maps are presented. For the rest of the integrated intensity maps, see Appendix A (Figs. A.1 and A.2). The kinematic analysis will be presented in an upcoming paper (Chacón-Tanarro et al., in prep., Paper II).

$\mathrm{CH}_{3} \mathrm{OH}$ and $\mathrm{CH}_{2} \mathrm{DOH}$. In Fig. 2, the integrated intensity map of the transitions $\mathrm{A}^{+}\left(2_{0,2}-1_{0,1}\right)$ of methanol and $\left(2_{0,2}-1_{0,1}\right)$ and $\left(3_{0,3}-2_{0,2}\right)$ of deuterated methanol are shown. Methanol, as previously reported, shows an asymmetric ringlike structure around the center of the core peaking toward the northeast at a distance of $4000 \mathrm{au}$. When the emission seen in methanol is averaged in concentric ellipses, as was done for the millimeter continuum maps in Chacón-Tanarro et al. (2017), it shows $\mathrm{CH}_{3} \mathrm{OH}$ depleted towards the center and enhanced at a distance of $\sim 3000$ au following a ring-like shape. The emission peak is shifted towards a more central region during this process (by $\sim 1000 \mathrm{au}$ ). This shift is with respect to the position of the methanol peak (as seen in Fig. 2), as the annular average includes regions with fainter $\mathrm{CH}_{3} \mathrm{OH}$ emission, which reduces the size of the ring compared to the distance of the $\mathrm{CH}_{3} \mathrm{OH}$ peak from the dust peak. The asymmetric distribution and the faint emission towards the south was already noticed by Spezzano et al. (2016), who showed that the $\mathrm{H}_{2}$ column density has a sharp drop toward the southern part of the core where photoprocesses dominate. The peak of the $\mathrm{CH}_{2} \mathrm{DOH}$ map extends toward the dust peak, suggesting that deuterated methanol may trace a higher density zone compared to the main isotopologue. We note that the clumpiness seen in the $\mathrm{CH}_{2} \mathrm{DOH}\left(2_{0,2}-1_{0,1}\right)$ map is due to noise (the noise in the integrated intensity is $0.005 \mathrm{~K} \mathrm{~km} \mathrm{~s}^{-1}$ ), and that the distribution of $\mathrm{CH}_{2} \mathrm{DOH}$ is better seen with the map of the $\left(3_{0,3}-2_{0,2}\right)$ line.

$\mathrm{H}_{2} \mathrm{CO}, \mathrm{H}_{2}^{13} \mathrm{CO}, \mathrm{HDCO}$, and $\mathrm{D}_{2} \mathrm{CO}$. The integrated intensity maps of $\mathrm{H}_{2}^{13} \mathrm{CO}\left(2_{1,2}-1_{1,1}\right)$, $\mathrm{HDCO}\left(2_{1,1}-1_{1,0}\right)$, and
$\mathrm{D}_{2} \mathrm{CO}\left(2_{1,2}-1_{1,1}\right)$ are shown in Fig. 3 , and the integrated intensity map of $\mathrm{H}_{2} \mathrm{CO}\left(2_{1,2}-1_{1,1}\right)$ is shown in Fig. A.2. The $\mathrm{H}_{2} \mathrm{CO}$ line is asymmetric and double-peaked, with the blue peak stronger than the red peak (see Fig. 1), suggestive of contraction motions, as already seen by Tafalla et al. (1998) and Bacmann et al. (2003). The $\mathrm{H}_{2}^{13} \mathrm{CO}\left(2_{1,2}-1_{1,1}\right)$ line matches with the absorption dip from the $\mathrm{H}_{2} \mathrm{CO}\left(2_{1,2}-1_{1,1}\right)$ line, indicating that this feature is indeed due to self-absorption (see Fig. 1). Thus, the distribution of formaldehyde is better traced by $\mathrm{H}_{2}^{13} \mathrm{CO}$, which also presents a ring-like structure around the core center with a peak close to the methanol peak and, unlike $\mathrm{CH}_{3} \mathrm{OH}$, a secondary maximum toward the south. However, $\mathrm{HDCO}$ and $\mathrm{D}_{2} \mathrm{CO}$ show centrally concentrated emission, differing from $\mathrm{CH}_{3} \mathrm{OH}, \mathrm{CH}_{2} \mathrm{DOH}$, and $\mathrm{H}_{2} \mathrm{CO}$.

$C^{17} \mathrm{O}$. The integrated intensity of $\mathrm{C}^{17} \mathrm{O}(1-0)$ is shown in Fig. 4. For the integrated intensity and the column density of $\mathrm{C}^{17} \mathrm{O}$, only the isolated component of the hyperfine structure $\left(F=\frac{5}{2}-\frac{5}{2}\right)$ is used. The distribution of $\mathrm{C}^{17} \mathrm{O}$ looks similar to the previous map presented by Caselli et al. (1999), although here the emission peak is more pronounced and the distribution is clumpier. These differences are caused by the different mapping methods: Caselli et al. (1999) only observed selected points, while we carried out OTF mapping of a more extended region. Interestingly, the emission peak coincides with the peak seen in $\mathrm{H}_{2}^{13} \mathrm{CO}$, as shown in Fig. 3. We point out that the $\mathrm{C}^{17} \mathrm{O}$ (1-0) transition is optically thin across the core, as already found by Caselli et al. (1999); this can also be seen in Fig. 1, where the relative intensities of the hyperfine components have been indicated below the observed spectrum.

\section{Analysis}

\subsection{Column densities}

All the column densities (see Sect. 5.1 for $\mathrm{H}_{2} \mathrm{CO}$ ) were derived assuming optically thin emission and assuming constant 

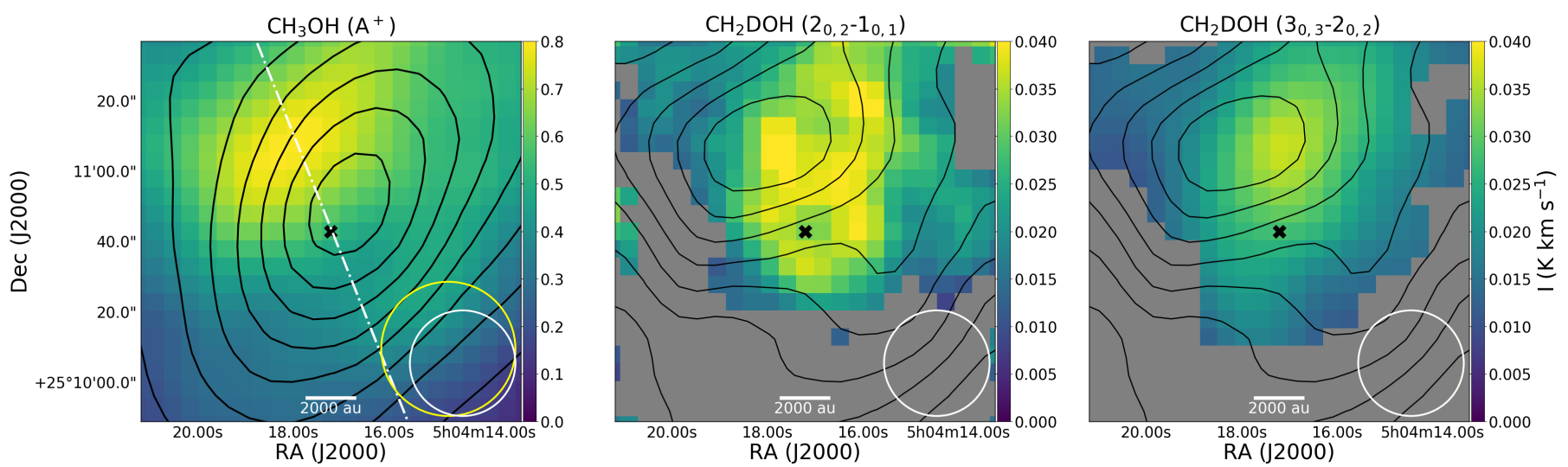

Fig. 2. Left panel: integrated intensity map of the strongest methanol transition observed $\left(2_{0,2}-1_{0,1}, \mathrm{~A}^{+}\right)$. The black contours represent increasing $10 \%$ steps of the $\mathrm{N}_{\mathrm{H}_{2}}$ column density map, derived by Spezzano et al. (2016) using Herschel/SPIRE data. The noise in the integrated intensity is $0.02 \mathrm{~K} \mathrm{~km} \mathrm{~s}^{-1}$. The white dash-dotted line indicates the cut used for comparison with the models (see Sect. 5). Middle panel: integrated intensity map of the deuterated methanol $\left(2_{0,2}-1_{0,1}\right)$ transition. The black contours represent increasing $10 \%$ steps of the $\mathrm{CH}_{3} \mathrm{OH}$ column density map, derived as explained in Sect. 4.1. The error on the integrated intensity is $0.005 \mathrm{~K} \mathrm{~km} \mathrm{~s}^{-1}$. Right panel: integrated intensity map of the deuterated methanol $\left(3_{0,3}-2_{0,2}\right)$ transition. The black contours represent increasing $10 \%$ steps of the $\mathrm{CH}_{3} \mathrm{OH}$ column density map, derived as explained in Sect. 4.1. The error on the integrated intensity is $0.004 \mathrm{~K} \mathrm{~km} \mathrm{~s}^{-1}$. In all panels the beams are shown in the bottom right corner of the figure, in yellow for Herschel/SPIRE and in white for the $30 \mathrm{~m}$ telescope; the black cross marks the dust continuum peak and only pixels with detection level above $3 \sigma$ are included.
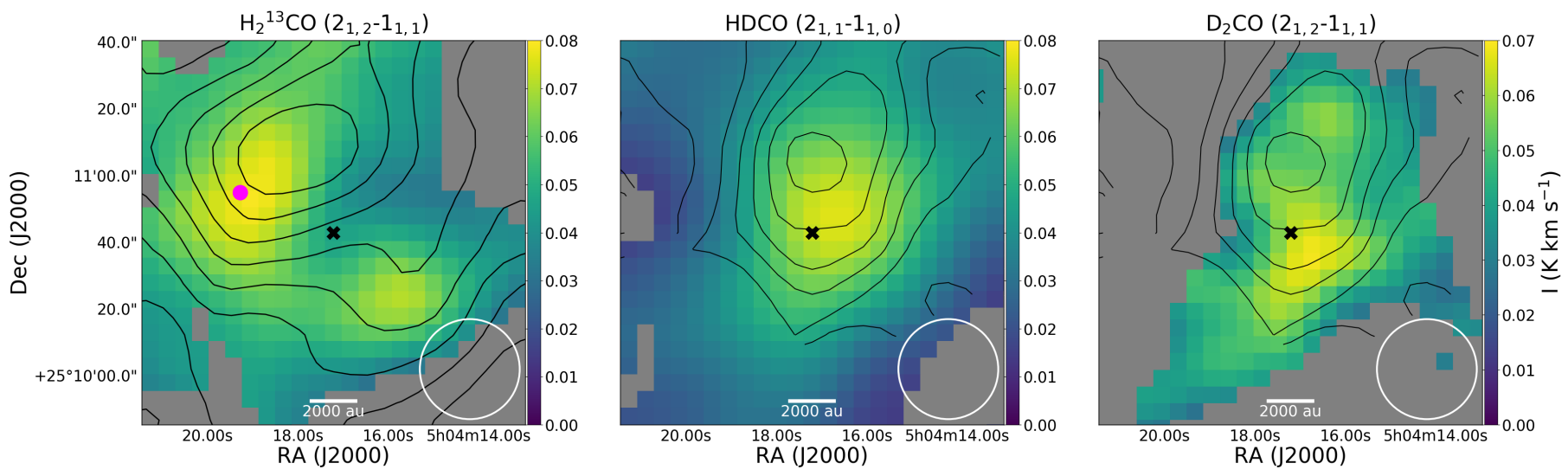

Fig. 3. Left panel: integrated intensity map of $\mathrm{H}_{2}^{13} \mathrm{CO}\left(2_{2,1}-1_{1,1}\right)$. The error on the integrated intensity is $0.01 \mathrm{~K} \mathrm{~km} \mathrm{~s}^{-1}$. The black contours represent increasing $10 \%$ steps of the $\mathrm{CH}_{3} \mathrm{OH}$ column density map, derived as explained in Sect. 4.1. The pink circle marks the $\mathrm{C}^{17} \mathrm{O}$ peak (see also Fig. 4). Middle panel: integrated intensity maps of $\operatorname{HDCO}\left(2_{1,1}-1_{1,0}\right)$. The error on the integrated intensity is $0.005 \mathrm{~K} \mathrm{~km} \mathrm{~s}^{-1}$. The black contours represent $10 \%$ steps with respect to the deuterated methanol column density peak. Right panel: integrated intensity map of $\mathrm{D}_{2} \mathrm{CO}\left(2_{1,2}-1_{1,1}\right)$. The error on the integrated intensity is $0.01 \mathrm{~K} \mathrm{~km} \mathrm{~s}^{-1}$. The black contours represent $10 \%$ steps with respect to the deuterated methanol column density peak. In all panels, the beam is shown in the bottom right corners, and the black cross marks the dust continuum peak. Only pixels with detection levels above $3 \sigma$ are included.

excitation temperature for all levels, following (Caselli et al. 2002b)

$N=\frac{8 \pi v^{3}}{c^{3}} \frac{Q\left(T_{\mathrm{ex}}\right)}{g_{u} A_{\mathrm{ul}}}\left[J_{v}\left(T_{\mathrm{ex}}\right)-J_{v}\left(T_{\mathrm{bg}}\right)\right]^{-1} \frac{\mathrm{e}^{\frac{E_{u}}{k T_{\mathrm{ex}}}}}{\mathrm{e}^{\frac{h v}{k T_{\mathrm{ex}}}}-1} \int T_{\mathrm{mb}} \mathrm{d} v$,

$Q\left(T_{\mathrm{ex}}\right)$ being the partition function of the molecule at an excitation temperature $T_{\mathrm{ex}}, g_{u}$ the rotational degeneracy of the upper level, $A_{\mathrm{ul}}$ the Einstein coefficient for spontaneous emission, $E_{u}$ the energy of the upper level, $J_{v}(T)$ the Rayleigh-Jeans Equivalent Temperature at the frequency $v$ and temperature $T$, and $T_{\mathrm{bg}}$ the temperature of the background, here the cosmic background $(2.7 \mathrm{~K})$.

Bizzocchi et al. (2014) obtained an excitation temperature for methanol of $T_{\mathrm{ex}}=6 \pm 3 \mathrm{~K}$, and derived the column density of deuterated methanol averaging the results for three different excitation temperatures within the same temperature range:
5 , 6.5, and $8 \mathrm{~K}$. The excitation temperature of $\mathrm{CH}_{2} \mathrm{DOH}$ cannot be derived because only two transitions were observed. Therefore, they calculated three column densities for $\mathrm{CH}_{2} \mathrm{DOH}$ using the three excitation temperatures to have an estimate of the uncertainty. Similarly, we derive the column density for both deuterated methanol lines assuming those three different excitation temperatures and averaging all the resulting column densities for the two lines. For methanol, the column density was derived in the same way using the two strongest lines observed $\left(\mathrm{A}^{+}\right.$and $\left.\mathrm{E}_{2}\right)$. Bizzocchi et al. (2014) derived an optical depth of $\tau<0.4$, and already demonstrated that considering optical depth effects does not vary significantly the resulting column density; thus, the assumption of optically thin emission is justified.

The values found for the column densities of $\mathrm{CH}_{2} \mathrm{DOH}$ and $\mathrm{CH}_{3} \mathrm{OH}$ are slightly higher than those found by Bizzocchi et al. (2014), but are within a factor of 2. The maps of $\mathrm{CH}_{3} \mathrm{OH}$ 


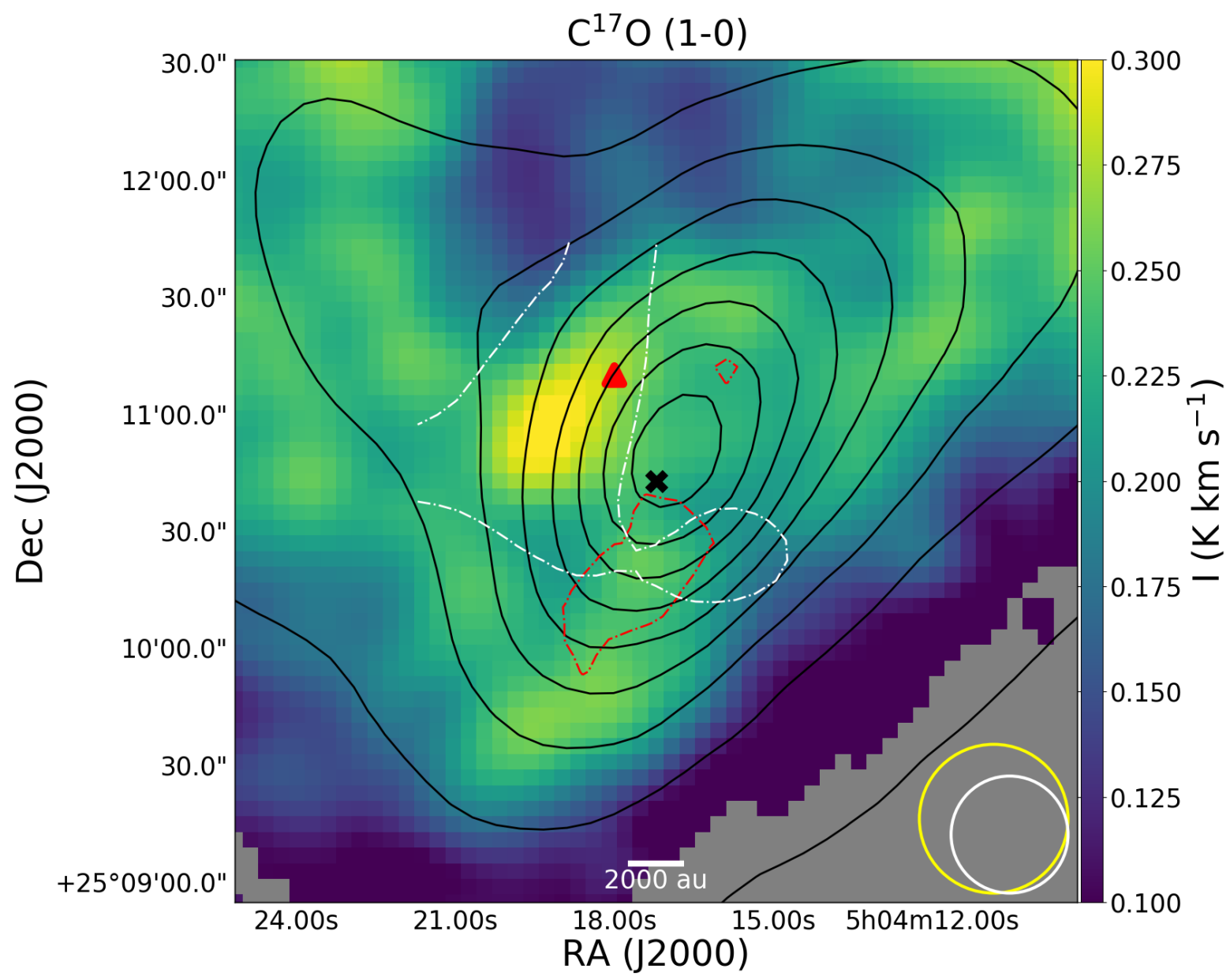

Fig. 4. Integrated intensity map of the $\mathrm{C}^{17} \mathrm{O}(1-0)$ line. The error on the integrated intensity is $0.02 \mathrm{~K} \mathrm{~km} \mathrm{~s}^{-1}$. The black contours represent increasing 10\% steps of the peak of the Herschel $\mathrm{N}\left(\mathrm{H}_{2}\right)$ map, presented by Spezzano et al. (2016); the white dash-dotted contour shows the $70 \%$ level of the $\mathrm{H}_{2}^{13} \mathrm{CO}\left(2_{2,1}-1_{1,1}\right)$ column density map, derived as explained in Sect. 4.1; and the red dash-dotted contour shows the $14 \sigma$ level $\left(\sigma=7 \mathrm{mK} \mathrm{km} \mathrm{s}^{-1}\right)$ of the integrated intensity of the $c-\mathrm{C}_{3} \mathrm{H}_{2}\left(3_{2,2}-3_{1,3}\right)$ line (Spezzano et al. 2016). The beams are shown in the bottom right corner, in yellow for Herschel/SPIRE and in white for the $30 \mathrm{~m}$ telescope. The black cross marks the dust continuum peak and the red triangle marks the methanol peak.

presented here are a factor of 3-20 noisier than the observations presented by Bizzocchi et al. (2014), due to the higher spectral resolution of these new maps. This can cause the difference seen in column densities. Nevertheless, the signal-to-noise ratio in these new maps is above $3 \sigma$ in the whole map for the two lines studied here, being $>20 \sigma$ towards the methanol peak.

For $\mathrm{C}^{17} \mathrm{O}$, the column density was derived assuming optically thin emission and a constant temperature of $10 \mathrm{~K}$ across the core. This temperature is based on Caselli et al. (1999), who obtained an excitation temperature of $10 \mathrm{~K}$ combining the emission of $\mathrm{C}^{17} \mathrm{O}(1-0)$ and $(2-1)$. The hyperfine structure was fitted to check possible optical-depth effects, finding a good match with the optically thin emission assumption, as previously reported by Caselli et al. (1999).

In the case of $\mathrm{H}_{2}^{13} \mathrm{CO}$, $\mathrm{HDCO}$, and $\mathrm{D}_{2} \mathrm{CO}$, a constant temperature of $7 \mathrm{~K}$ was used. This temperature comes from the modeling done for $\mathrm{H}_{2} \mathrm{CO}$ (see Sect. 5.1), and is in agreement with that used by Bacmann et al. (2003). The line $\mathrm{D}_{2} \mathrm{CO}\left(2_{1,2}-1_{1,1}\right)$ is due to para species, so to obtain the total column density of $\mathrm{D}_{2} \mathrm{CO}$, the corresponding energies, degeneracy, and the total partition function were used assuming an ortho-to-para ratio of $2: 1$. This same procedure was applied to $\mathrm{H}_{2}^{13} \mathrm{CO}\left(2_{1,2}-1_{1,1}\right)$, which is due to ortho species, assuming an ortho-to-para ratio of $3: 1$. The column density map of $\mathrm{H}_{2}^{13} \mathrm{CO}$ is used to derive a column density map of $\mathrm{H}_{2} \mathrm{CO}$ assuming $\left[{ }^{12} \mathrm{C}\right] /\left[{ }^{13} \mathrm{C}\right]=77$ (Wilson \& Rood 1994).

All this information, together with the spectroscopic parameters used in the column density derivations, are summarized in Table 2. The resulting column density maps can be found in Appendix B (Figs. B.1-B.6). Here we only present the values at the dust and methanol peaks shown in Table 3. We note that in this table the value for the column density of $\mathrm{H}_{2} \mathrm{CO}$ is the one derived in Sect. 5.1.

We note that the $E_{u}$ and $A$ values (see Table 2) of the different molecules are similar, and therefore, the errors associated with the assumptions of the above excitation temperatures are within the errors associated with the noise of the data. This can also be seen from the error of the column densities of $\mathrm{CH}_{2} \mathrm{DOH}$ and $\mathrm{CH}_{3} \mathrm{OH}$ (see Table 3). These errors have been derived from the maximum spread of column density values found for the three different excitation temperatures and the two lines used for each species. The adopted assumption on the excitation temperature does not bias our results significantly because said errors are $\lesssim 25 \%$ of the column density values. Nevertheless, further observations are needed to improve the determination of the excitation temperatures. 
Table 2. Parameters used in the derivation of the column densities.

\begin{tabular}{cccccc}
\hline \hline Line & $T_{\text {ex }}(\mathrm{K})$ & $Q\left(T_{\mathrm{ex}}\right)$ & $E_{u} / k_{b}(\mathrm{~K})$ & $A\left(10^{-5} \mathrm{~s}^{-1}\right)$ & $g_{u}$ \\
\hline $\mathrm{CH}_{3} \mathrm{OH}\left(\mathrm{A}^{+}\right)$ & $5,6.5,8$ & $5.46,10.09,15.37$ & 6.96 & 0.34 & 5 \\
$\mathrm{CH}_{3} \mathrm{OH}\left(\mathrm{E}_{2}\right)$ & $5,6.5,8$ & $5.46,10.09,15.37$ & 12.53 & 0.256 & 5 \\
$\mathrm{CH}_{2} \mathrm{DOH}\left(2_{0,2}-1_{0,1}\right)$ & $5,6.5,8$ & $9.60,15.25,22.45$ & 6.40 & 0.202 & 5 \\
$\mathrm{CH}_{2} \mathrm{DOH}\left(3_{0,3}-2_{0,2}\right)$ & $5,6.5,8$ & $9.60,15.25,22.45$ & 12.83 & 0.730 & 7 \\
$\mathrm{H}_{2}^{13} \mathrm{CO}\left(2_{1,2}-1_{1,1}\right)$ & 7 & 8.95 & 21.72 & 4.931 & 15 \\
$\mathrm{HDCO}\left(2_{1,1}-1_{1,0}\right)$ & 7 & 7.67 & 12.23 & 4.591 & 5 \\
$\mathrm{D}_{2} \mathrm{CO}\left(2_{1,2}-1_{1,1}\right)$ & 7 & 15.64 & 13.37 & 2.583 & 5 \\
$\mathrm{C}^{17} \mathrm{O}(1-0)$ & 10 & 24.37 & 5.39 & 0.0067 & 6 \\
\hline
\end{tabular}

Notes. Columns are: excitation temperature and the corresponding partition function, the energy of the upper levels of the transitions relative to the ground states, the Einstein coefficients and the degeneracy of the upper levels. For the case of $\mathrm{C}^{17} \mathrm{O}$ these parameters correspond to the isolated hyperfine component. The references for these spectroscopic parameters can be found in Table 1.

Table 3. Column densities of the molecules observed.

\begin{tabular}{ccc}
\hline \hline Line & $\begin{array}{c}N_{\text {Dust peak }} \\
\left(10^{12} \mathrm{~cm}^{-2}\right)\end{array}$ & $\begin{array}{c}N_{\text {Methanol peak }} \\
\left(10^{12} \mathrm{~cm}^{-2}\right)\end{array}$ \\
\hline $\mathrm{CH}_{3} \mathrm{OH}$ & $39 \pm 4$ & $59 \pm 6$ \\
$\mathrm{CH}_{2} \mathrm{DOH}$ & $2.8 \pm 0.7$ & $3.3 \pm 0.8$ \\
$\mathrm{H}_{2} \mathrm{CO}$ & $9 \pm 2$ & $7 \pm 3$ \\
$\mathrm{H}_{2}^{13} \mathrm{CO}$ & $0.54 \pm 0.35$ & $0.78 \pm 0.35$ \\
$\mathrm{HDCO}$ & $1.30 \pm 0.09$ & $0.96 \pm 0.09$ \\
$\mathrm{D}_{2} \mathrm{CO}$ & $1.5 \pm 0.3$ & $1.1 \pm 0.3$ \\
$\mathrm{C}^{17} \mathrm{O}$ & $680 \pm 60$ & $810 \pm 60$ \\
\hline
\end{tabular}

Notes. The value derived for $\mathrm{H}_{2} \mathrm{CO}$ comes from the average between the results of two radiative transfer models, as described in Sect. 5.1, and the error is the difference between the two values.

\subsection{Deuterium fraction}

\subsection{1. $\mathrm{CH}_{2} \mathrm{DOH} / \mathrm{CH}_{3} \mathrm{OH}$}

The deuterium fraction map of methanol was obtained by taking the ratio between the column density of $\mathrm{CH}_{2} \mathrm{DOH}$ and that of $\mathrm{CH}_{3} \mathrm{OH}$. Figure 5 shows a nonsymmetric distribution peaking toward the south but close to the dust peak, especially when the beam size is taken into account (see Fig. C.1. for the uncertainties). The $\left[\mathrm{CH}_{2} \mathrm{DOH}\right] /\left[\mathrm{CH}_{3} \mathrm{OH}\right]$ peak value, $\sim 0.08 \pm 0.02$, is consistent with that found by Bizzocchi et al. (2014) toward the core center.

\subsection{2. $\mathrm{H}_{2} \mathrm{CO}, \mathrm{HDCO}$, and $\mathrm{D}_{2} \mathrm{CO}$}

The deuterium fraction maps of formaldehyde are shown in Fig. 6. They are derived using the column density maps of $\mathrm{HDCO}, \mathrm{D}_{2} \mathrm{CO}$, and $\mathrm{H}_{2} \mathrm{CO}$. The $\mathrm{H}_{2} \mathrm{CO}$, as commented previously, is derived from the map of the optically thin emission of $\mathrm{H}_{2}^{13} \mathrm{CO}$. The maps show that the deuteration of formaldehyde also occurs in the central regions of the core. However, this needs more sensitive observations because of the large uncertainties, which can be seen in Fig. C.2. Thus, no information regarding the distribution can be obtained. As reference, the values at the dust peak are $[\mathrm{HDCO}] /\left[\mathrm{H}_{2} \mathrm{CO}\right] \simeq 0.03 \pm 0.02$ and $\left[\mathrm{D}_{2} \mathrm{CO}\right] /\left[\mathrm{H}_{2} \mathrm{CO}\right] \simeq 0.04 \pm 0.03$.

The map of the $\left[\mathrm{D}_{2} \mathrm{CO}\right] /[\mathrm{HDCO}]$ ratio shows a very high level of deuteration, with a mean value of $\sim 1.2 \pm 0.3$. As previously mentioned, any variation in the deuteration across the map

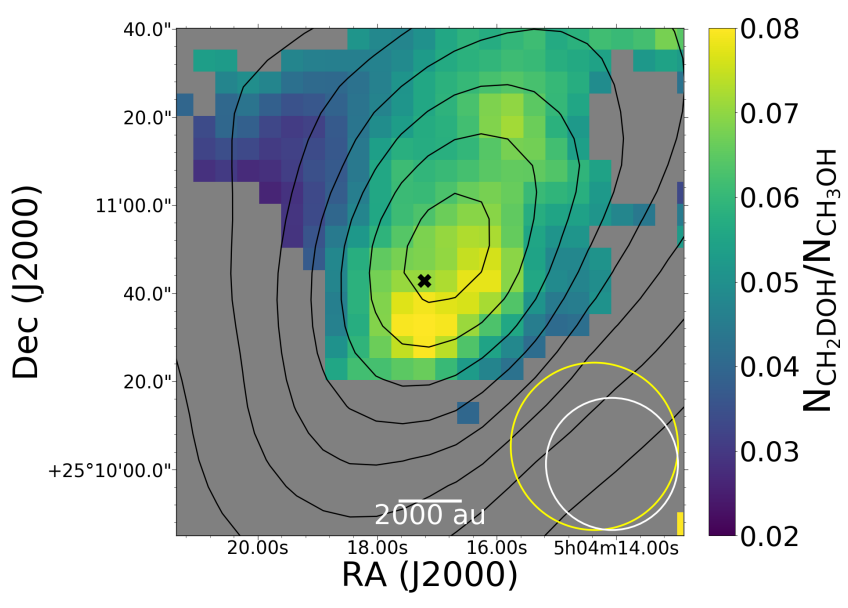

Fig. 5. Deuterium fraction map of methanol using the column density maps of $\mathrm{CH}_{2} \mathrm{DOH}$ and $\mathrm{CH}_{3} \mathrm{OH}$ derived as described in Sect. 4.1. The black contours represent increasing $10 \%$ steps of the peak of the Herschel $\mathrm{N}\left(\mathrm{H}_{2}\right)$ map, presented by Spezzano et al. (2016). The beams are shown in the bottom right corner of the figure, in yellow for Herschel/SPIRE and in white for the $30 \mathrm{~m}$ telescope. The black cross marks the dust continuum peak, and only values above the $3 \sigma$ detection level are considered.

is not significant, due to the high errors in its determination (see Fig. C.2).

\section{Comparison with models}

In this section, observational and theoretical results are compared. Here we present two different chemical models applied to L1544, and use them to compare the modeled column densities with the observed values and to estimate the $\mathrm{H}_{2} \mathrm{CO}$ column density.

S16. In this case the abundances of $\mathrm{C}^{17} \mathrm{O}, \mathrm{CH}_{3} \mathrm{OH}$, $\mathrm{CH}_{2} \mathrm{DOH}, \mathrm{H}_{2} \mathrm{CO}$, HDCO, and $\mathrm{D}_{2} \mathrm{CO}$ were calculated using the chemical model presented in Sipilä et al. (2015a,b), which includes extensive descriptions of deuterium and spin-state chemistry. The modeling process was similar to that discussed in Sipilä et al. (2016), i.e., we divided the L1544 core model presented by Keto \& Caselli (2010; see also Keto et al. 2014) into concentric shells, calculated the chemistry separately in each shell, and extracted the abundance gradients of the various species at several time steps. The model uses a bulk ice model, and it includes a fixed reactive desorption efficiency. The results 

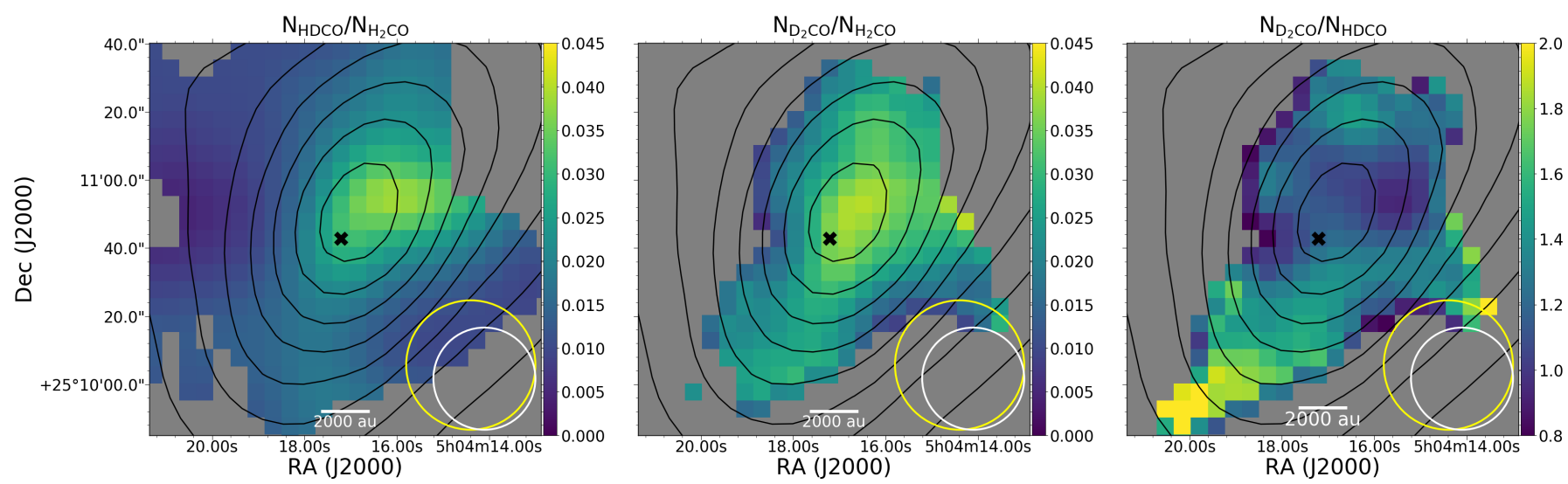

Fig. 6. Left panel: $[\mathrm{HDCO}] /\left[\mathrm{H}_{2} \mathrm{CO}\right]$ map using the column density maps of $\mathrm{HDCO}$ and $\mathrm{H}_{2} \mathrm{CO}$ derived as described in Sect. 4.1. Middle panel: map of $\left[\mathrm{D}_{2} \mathrm{CO}\right] /\left[\mathrm{H}_{2} \mathrm{CO}\right]$, using the column density maps of $\mathrm{D}_{2} \mathrm{CO}$ and $\mathrm{H}_{2} \mathrm{CO}$ derived as described in Sect. 4.1. Right panel: map of the ratio $\left[\mathrm{D}_{2} \mathrm{CO}\right] /[\mathrm{HDCO}]$, using the column density maps of $\mathrm{HDCO}$ and $\mathrm{D}_{2} \mathrm{CO}$ derived as described in Sect. 4.1. In all panels the black contours represent increasing 10\% steps of the peak of the Herschel $\mathrm{N}_{\mathrm{H}}$ ) map, presented by Spezzano et al. (2016). The beams are shown in the bottom right corners of the figures, in yellow for Herschel/SPIRE and in white for the $30 \mathrm{~m}$ telescope. The black cross marks the dust continuum peak, and only values above the $3 \sigma$ detection level are considered.
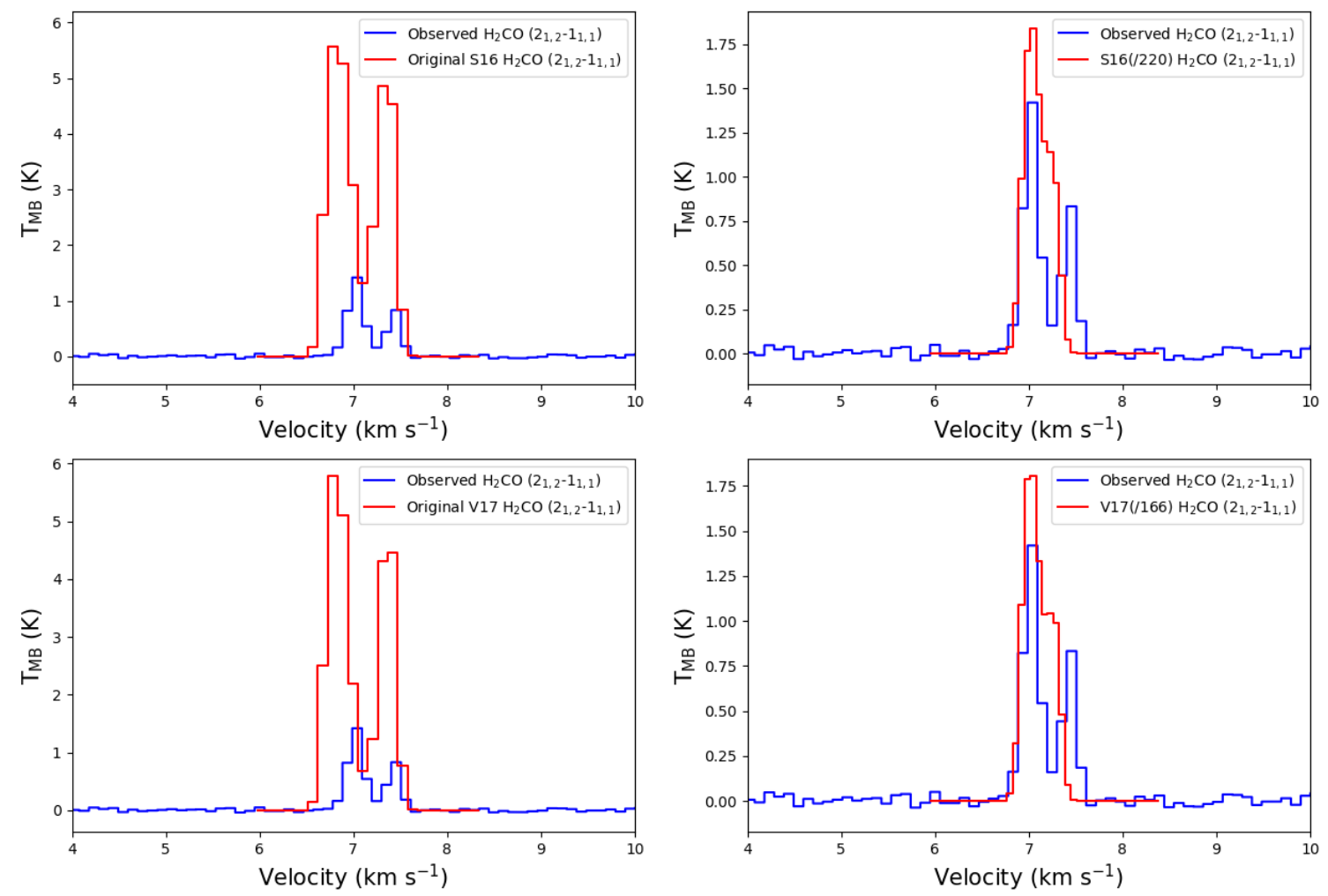

Fig. 7. Comparison of the emission modeled by MOLLIE (in red, see Sect. 5.1) and the observed emission (in blue) of the $\mathrm{H}_{2} \mathrm{CO}$ transition observed at the center of the core. Top panels: results using S16 abundance profile (left panel), and the same scaled by a factor of 220 (right panel). Bottom panels: results using V17 abundance profile (left panel), and the same scaled by a factor of 166 (right panel). The original abundance profiles overestimate the emission of the line by a factor of $\sim 3$, and the scaled abundance profiles do not reproduce the shape of the line.

shown in Figs. 7-11 correspond to the time when the CO column density is comparable to the observed value in L1544 (Caselli et al. 1999).

V17. The model of the pre-stellar core L1544 by Vasyunin et al. (2017) utilized static 1D distributions of gas density, and gas and dust temperatures from Keto et al. (2014). The model includes time-dependent description of gas and grain chemistry based on Vasyunin \& Herbst (2013) with several major updates concerning new gas-phase reactions important for the formation of complex organic molecules in the cold gas, as well as the detailed treatment of the efficiency of reactive desorption based on experimental works by Minissale et al. (2016). Treatment of chemistry on interstellar grains is based on a multilayer approach to the structure of icy mantles, which allows us to discriminate between the reactive surface of ice and more chemically inert ice bulk. Since the temperature in L1544 is $\sim 10 \mathrm{~K}$, the only important source of mobility of species on ice surfaces is quantum tunneling, which is enabled for $\mathrm{H}$ and $\mathrm{H}_{2}$. 


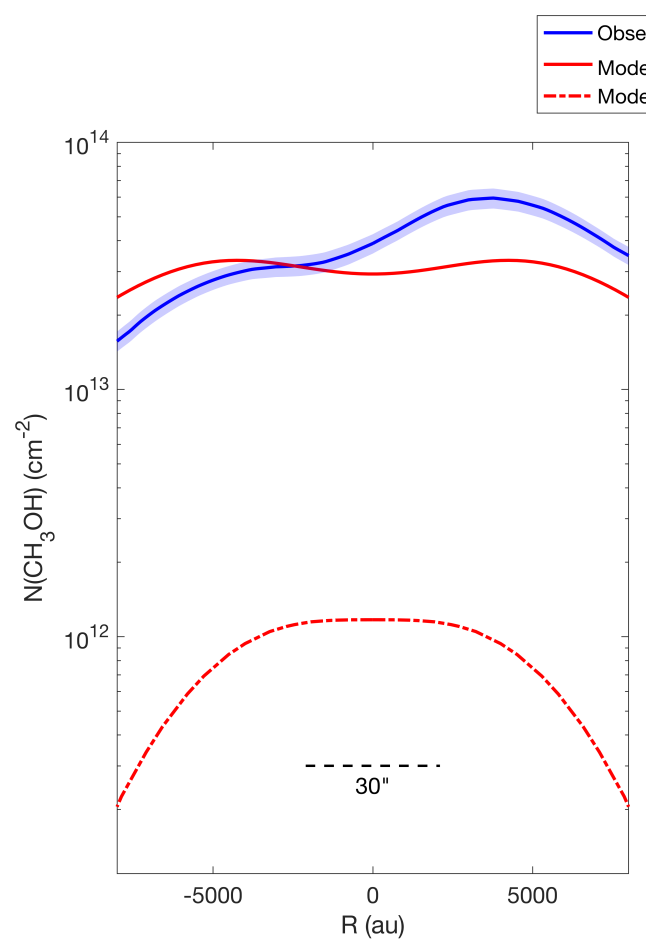

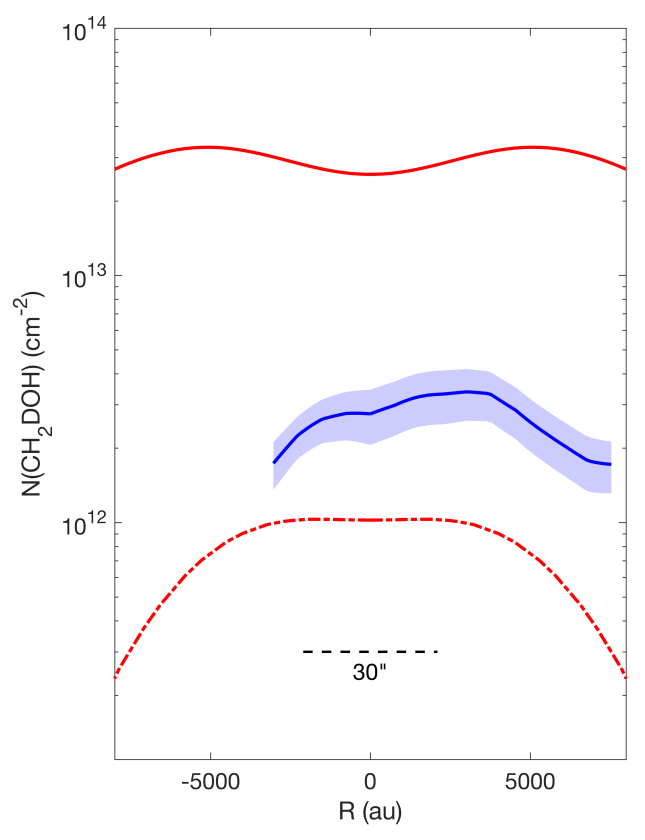

Fig. 8. Comparison of the observed column densities (blue line) with the modeled column densities by V17 (red line) and $\mathrm{S} 16$ (red dashed line) of $\mathrm{CH}_{3} \mathrm{OH}$ (left panel) and $\mathrm{CH}_{2} \mathrm{DOH}$ (right panel). The observed column density profiles are taken along the white dashed line shown in Fig. 2, being $R=0$ au the dust peak, $R>0$ au the direction from dust peak to methanol peak, and $R<0$ au the direction from the dust peak toward the southwest. The shaded blue regions indicate the error bars of the column densities. The resolution is $30^{\prime \prime}$, shown at the bottom of the figure. A scaling factor has been applied to the models.
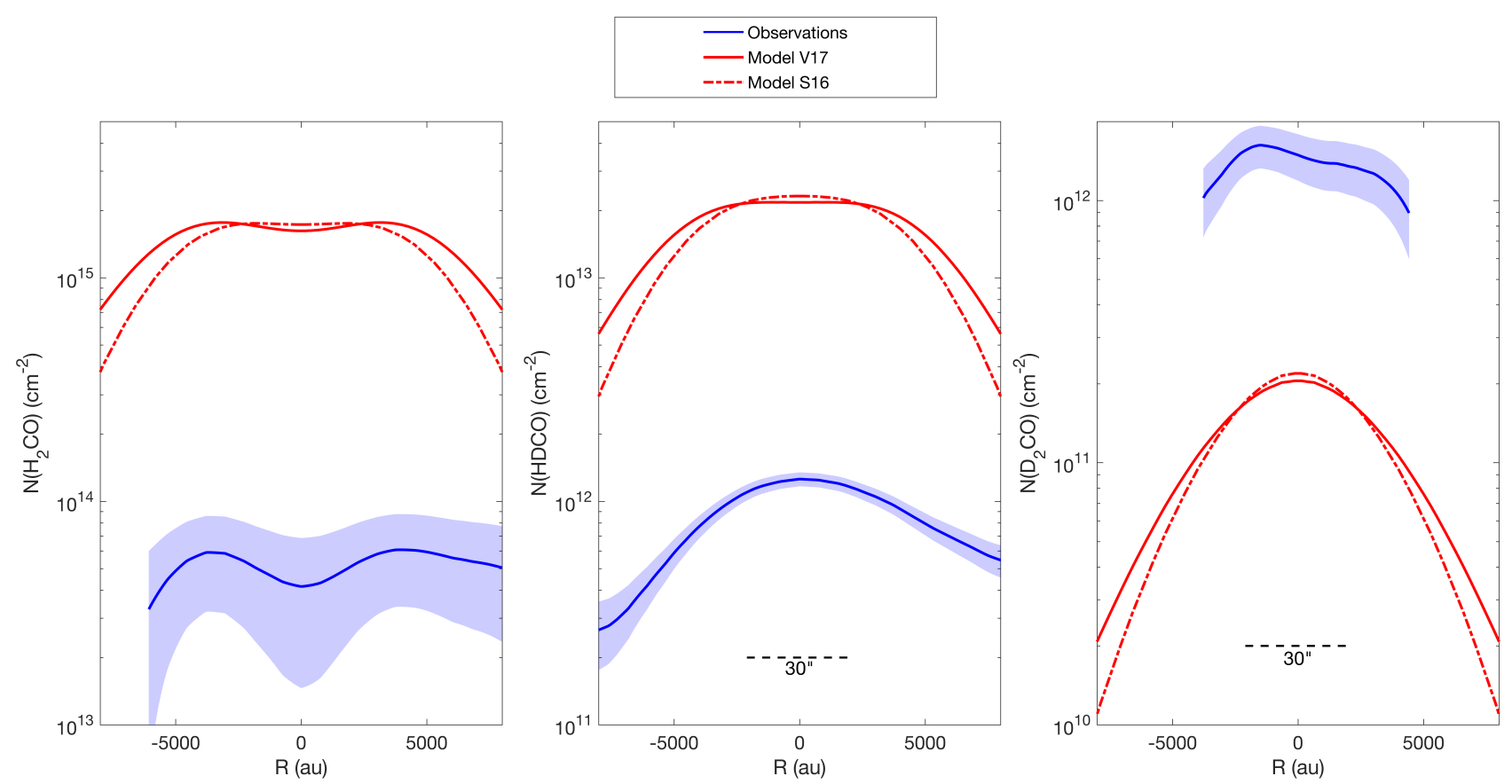

Fig. 9. Comparison of the observed column densities (blue line) with the modeled column densities by V17 (red line) and S16 (red dashed line) of $\mathrm{H}_{2} \mathrm{CO}$ (left panel), $\mathrm{HDCO}$ (middle panel), and $\mathrm{D}_{2} \mathrm{CO}$ (right panel). The observed column density profiles are taken along the white dashed line shown in Fig. 2, being $R=0$ au the dust peak, $R>0$ au the direction from dust peak to methanol peak, and $R<0$ au the direction from the dust peak toward the southwest. The shaded blue regions indicate the error bars of the column densities. The resolution is $30^{\prime \prime}$, shown at the bottom of the figure.

Both $\mathrm{H}_{2} \mathrm{CO}$ and $\mathrm{CH}_{3} \mathrm{OH}$ in the models are produced on the surface in the hydrogenation sequence of $\mathrm{CO}$ :

$\mathrm{CO} \rightarrow \mathrm{HCO} \rightarrow \mathrm{H}_{2} \mathrm{CO} \rightarrow \mathrm{CH}_{2} \mathrm{OH} \rightarrow \mathrm{CH}_{3} \mathrm{OH}$.

However, while the surface route is the only efficient way of formation for $\mathrm{CH}_{3} \mathrm{OH}, \mathrm{H}_{2} \mathrm{CO}$ is also formed efficiently in the gas-phase reaction

$\mathrm{O}+\mathrm{CH}_{3} \rightarrow \mathrm{H}_{2} \mathrm{CO}+\mathrm{H}$.
In summary, both models use the same physical cloud structure, do not follow the evolution of the density and temperature of the cloud with time (here called static models), and adopt the same chemical reactions. However, the S16 model uses a bulk ice model with a fixed reactive desorption efficiency, it includes deuterated species, allows diffusion of light species via thermal hopping, and starts its chemistry from atomic initial abundances. Meanwhile, V17 does not include deuterated isotopologues, but uses a multilayer approach for the ices with an updated treatment 


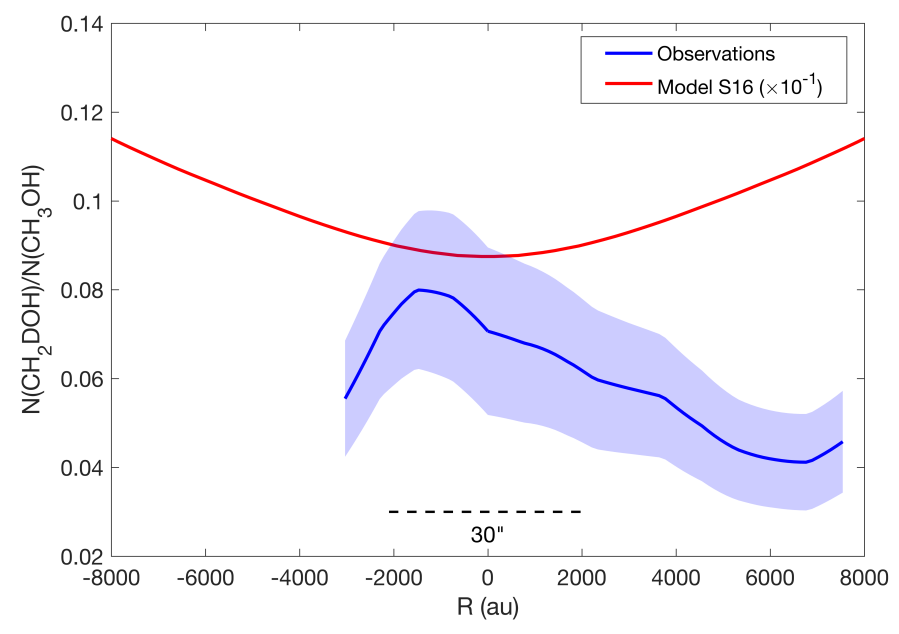

Fig. 10. Comparison of the observed methanol deuterium fraction (blue line) with the modeled deuterium fraction by S16 (red line). The observed deuteration profile is taken along the white dashed line in Fig. 2, being $R=0$ au the dust peak, $R>0$ au the direction from dust peak to methanol peak, and $R<0$ au the direction from the dust peak toward the southwest. The shaded blue region shows the error bars in the deuterium fractions. The resolution is $30^{\prime \prime}$, shown at the bottom of the figure. A scaling factor has been applied to the model.

of the reactive desorption efficiency, and also allows hydrogen atoms and molecules to quantum tunnel on the grain surfaces, which facilitates the formation of methanol and other complex organic molecules (COMs). Moreover, this model assumes the evolved chemistry of diffuse clouds as initial conditions. However, no matter what initial conditions we use (i.e., if we start our calculation with atomic conditions, typical of diffuse clouds, or if we start with conditions typical of molecular clouds), the final modeled results for our pre-stellar core chemical composition are the same. Therefore, different initial conditions do not play a role in the different results from the models concerning methanol; the differences here can solely be ascribed to the different diffusion mechanisms of $\mathrm{H}$ on the surface, with V17 allowing quantum tunneling and S16 allowing the significantly slower thermal hopping. The comparison between models is important to check the dominant processes in the formation of COMs.

\subsection{Deriving the column density of $\mathrm{H}_{2} \mathrm{CO}$}

As already stated, $\mathrm{H}_{2} \mathrm{CO}$ presents signatures of self-absorption, so we cannot apply Eq. (1) and the assumption of optical thinness to this molecule. Thus, we modeled the emission at the center of the core via radiative transfer modeling using MOLLIE (Keto \& Rybicki 2010), the physical model for L1544 from Keto et al. (2015), and the abundances profiles obtained from S16 and V17. The resulting column densities from MOLLIE were smoothed with a $30^{\prime \prime}$ beam to match the resolution of the observations. This procedure was only used to compare the column density derived from modeling with the observed values derived from the $\mathrm{H}_{2}^{13} \mathrm{CO}$ line.

We found that both abundance profiles overestimated the emission by a factor of $\sim 3$ (see Fig. 7). Thus, we scaled the abundance profiles to reproduce the observed peak emission. The scaling factor applied to the abundance profile of V17 was 166 , while the abundance profile from S16 needed to be reduced by a factor 220 . The fact that the abundances had to be reduced by such large factors is due to the high optical depth of the line. The resulting modeled column densities can be found in Table 3 .
The excitation temperature obtained in both cases was $\sim 7 \mathrm{~K}$ in the central $0.1 \mathrm{pc}$, and this value has been adopted as the temperature used for the derivation of the $\mathrm{HDCO}, \mathrm{D}_{2} \mathrm{CO}$, and $\mathrm{H}_{2}^{13} \mathrm{CO}$ column densities. At the center of the core the column density of $\mathrm{H}_{2} \mathrm{CO}$ derived from $\mathrm{H}_{2}^{13} \mathrm{CO}$ is a factor of 5 higher than the column density derived here using MOLLIE. This difference is below $2 \sigma$ (being $\sigma$ the error on column density derived observationally) once the uncertainties are taken into account.

The abundance reduction produces a change in the line profile, which now does not show the observed double peaked asymmetry any longer (see Fig. 7). One explanation is that the models do not take into account the presence of a lower density molecular material surrounding the core, where $\mathrm{H}_{2} \mathrm{CO}$ can be present and participate in the line absorption. In fact, the results from the $\mathrm{H}_{2}^{13} \mathrm{CO}$ line suggest an optical depth of $\tau \sim 1.4$ for the $\mathrm{H}_{2} \mathrm{CO}$ line, while the optical depth predicted by MOLLIE of the $\mathrm{H}_{2} \mathrm{CO}$ line is $\tau \sim 0.3$. The ones presented here are therefore only approximate estimates of the column density, and although they are not far from the observations, more detailed work (i.e., larger scale mapping of $\mathrm{H}_{2} \mathrm{CO}$ ) is needed to fully understand from which layer of the cloud the formaldehyde emission is coming.

\subsection{Modeled vs. observed column densities}

In this section, the column densities observed are compared with the results from the V17 and S16 models. The V17 model does not include deuterated species so in this case the modeled column densities of deuterated species were derived using the column density of their corresponding isotopologue and the deuteration ratio from $\mathrm{S} 16$.

The results can be found in Fig. 8 for methanol and deuterated methanol and in Fig. 9 for single and doubly deuterated formaldehyde (we note the scaling factor applied to the models in some figures, indicated in the legend). The observed column densities are those corresponding to a cut done in the maps, which follows the white dashed line showed in Fig. 2. This cut is the same as the one studied by Spezzano et al. (2016) who considered the variation in the methanol column density from the methanol peak to the dust peak. We point out that the same conclusions are reached if the cut orientation is changed, as the methanol column density changes by a factor of a few around the asymmetric ring, while the difference with the model prediction is larger than one order of magnitude.

Methanol and deuterated methanol are overestimated in the model from $\mathrm{V} 17$, and underestimated by $\mathrm{S} 16$. The overproduction of $\mathrm{CH}_{3} \mathrm{OH}$ in $\mathrm{V} 17$, which was already noted by the authors, can be due to an overestimation of the efficiency of reactive desorption. Moreover, as explained in V17, this model does not efficiently form $\mathrm{CO}_{2}$ because of the activation energy of the $\mathrm{CO}+\mathrm{OH}$ reaction (Ruffle \& Herbst 2001), which does not proceed at $T<20 \mathrm{~K}$ (Vasyunin et al. 2017), so in warmer environments, we would expect to have a significant fraction of $\mathrm{CO}$ producing $\mathrm{CO}_{2}$ instead of methanol. $\mathrm{CO}_{2}$ is also abundant and ubiquitous in quiescent dense clouds (Whittet et al. 1998; Boogert et al. 2015); however, in these cold regions, $\mathrm{CO}_{2}$ is expected to form via energetic processing of water ice mantles onto carbonaceous grains (see, e.g., Mennella et al. 2004, 2006), and this process has not been included in any gas-grain chemical model yet. In any case, we note that a factor of 10 difference between model and observations can be considered to be in fair agreement, due to the large uncertainties of gas-grain models, which can go up to one order of magnitude (Vasyunin et al. 2004, 2008). The underproduction in S16 is most likely caused 

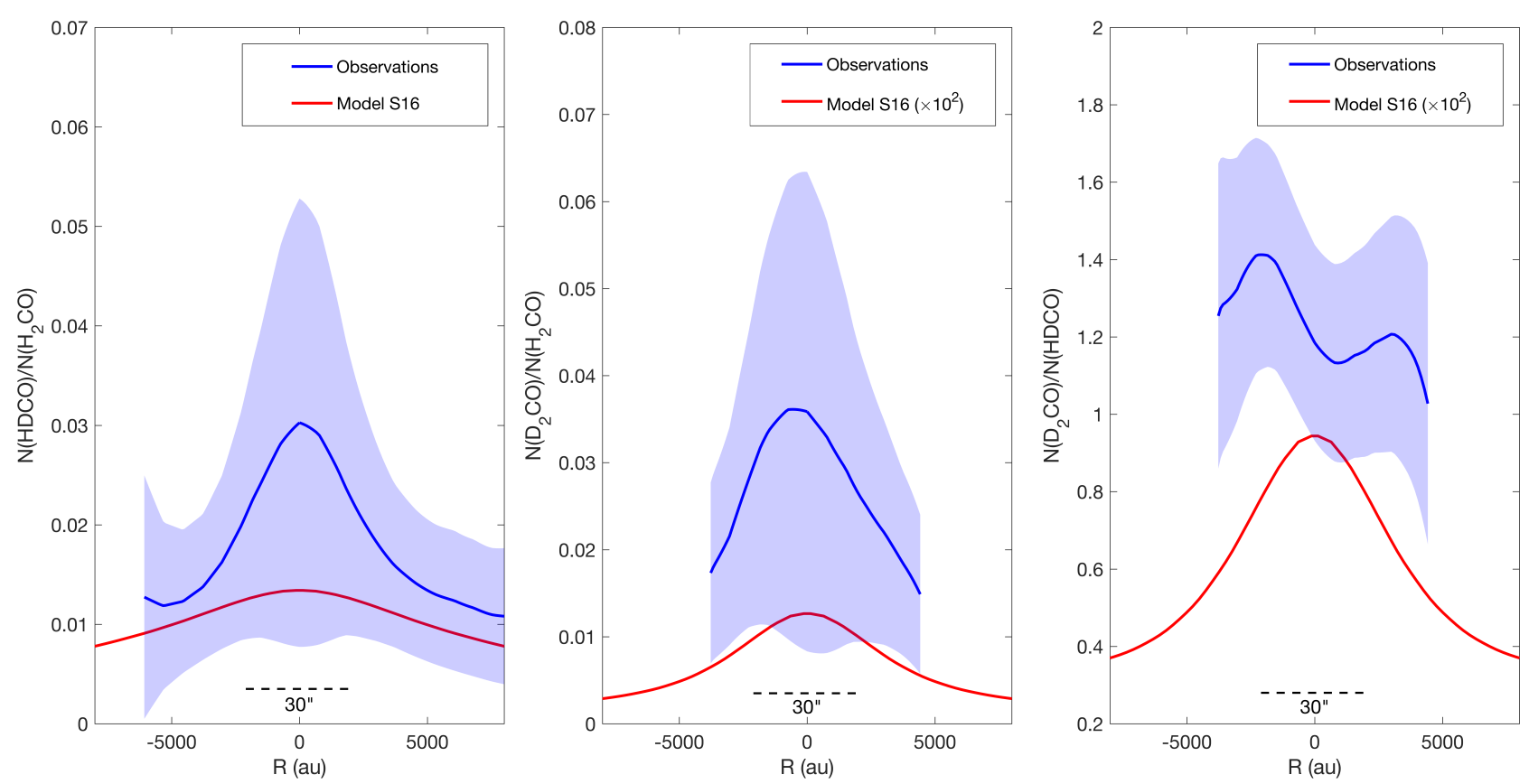

Fig. 11. Comparison of the observed $[\mathrm{HDCO}] /\left[\mathrm{H}_{2} \mathrm{CO}\right]$ (left panel), $\left[\mathrm{D}_{2} \mathrm{CO}\right] /\left[\mathrm{H}_{2} \mathrm{CO}\right]$ (middle panel), and $\left[\mathrm{D}_{2} \mathrm{CO}\right] /[\mathrm{HDCO}]$ (right panel) ratios (blue line) with the modeled deuterium fractions by S16 (red line). The observed deuteration profile is taken along the white dashed line in Fig. 2 , being $R=0$ au the dust peak, $R>0$ au the direction from dust peak to methanol peak, and $R<0$ au the direction from the dust peak toward the southwest. The shaded blue regions indicate the error bars in the deuterium fractions. The resolution is $30^{\prime \prime}$, shown at the bottom of the figure. A scaling factor has been applied to the models.

by the fact that hydrogen atoms are not allowed to quantum tunnel across the grain surface, thus significantly reducing surface hydrogenation and the consequent production of $\mathrm{CH}_{3} \mathrm{OH}$.

The measured column density of methanol is closer to the V17 predictions, whereas deuterated methanol is closer to S16. It should be taken into account that the deuterium fraction for the models was taken from S16, which overestimates the deuteration of methanol by a factor of 10 (see Fig. 10). If the observed deuterium fraction $(\sim 0.1)$ is applied to the models, the difference between observations and the two models is similar.

In the case of formaldehyde, both models overproduce $\mathrm{H}_{2} \mathrm{CO}$. For its deuteration, the $\mathrm{S} 16$ model predicts $[\mathrm{HDCO}] /\left[\mathrm{H}_{2} \mathrm{CO}\right] \sim 0.013$, and $\left[\mathrm{D}_{2} \mathrm{CO}\right] /\left[\mathrm{H}_{2} \mathrm{CO}\right]$ a factor of 100 smaller (see Fig. 11). However, observationally, we found a value of $\sim 0.03 \pm 0.02$ for $[\mathrm{HDCO}] /\left[\mathrm{H}_{2} \mathrm{CO}\right]$ and $\sim 0.04 \pm 0.03$ for $\left[\mathrm{D}_{2} \mathrm{CO}\right] /\left[\mathrm{H}_{2} \mathrm{CO}\right]$. This means that while $[\mathrm{HDCO}] /\left[\mathrm{H}_{2} \mathrm{CO}\right]$ is reproduced well by the model, there is a difference of a factor of 100 between the observed $\left[\mathrm{D}_{2} \mathrm{CO}\right] /\left[\mathrm{H}_{2} \mathrm{CO}\right]$ and the modeled ratio. Applying this observed factor to the modeled abundances of $\mathrm{H}_{2} \mathrm{CO}$ would imply an overproduction of the whole formaldehyde family (see Fig. 9).

Our results show that the production of these molecules is still uncertain in this core, and more work has to be done to better constrain the chemistry in pre-stellar cores.

\section{Discussion}

\subsection{Distribution}

The fact that deuterated methanol peaks closer to the core center compared to methanol can be simply explained by the larger $\mathrm{D} / \mathrm{H}$ abundance, so that $\mathrm{D}$ atoms compete with $\mathrm{H}$ atoms in saturating solid CO. This central region is in fact characterized by large amounts of CO freeze-out (Caselli et al. 1999) and consequently large deuterium fractions (Caselli et al. 2002b, 2003; Crapsi et al.

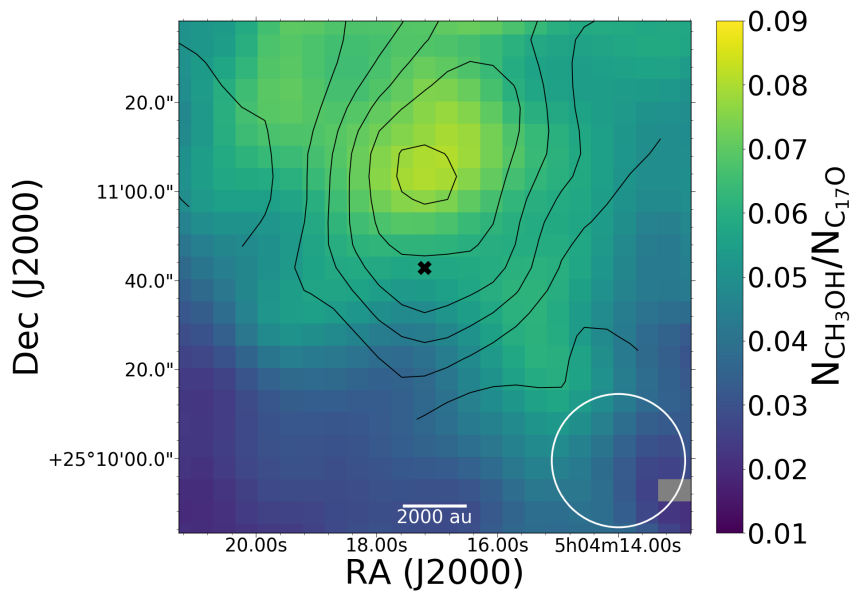

Fig. 12. Ratio of the column densities of methanol to $\mathrm{C}^{17} \mathrm{O}$. The black contours represent $10 \%$ steps with respect to the deuterated methanol column density peak. The black cross marks the dust peak. The beam is shown in the bottom right corner.

2007), as predicted by theory (Dalgarno \& Lepp 1984; Walmsley et al. 2004). This scenario is supported by the fact that deuterated methanol peaks exactly where the $\mathrm{N}\left(\mathrm{CH}_{3} \mathrm{OH}\right) / \mathrm{N}(\mathrm{CO})$ ratio is higher (see Fig. 12), and the deuterium fraction is enhanced where the $\mathrm{CO}$ is more depleted (see Fig. B.6).

For formaldehyde, we find that it follows a slightly different distribution compared to $\mathrm{CH}_{3} \mathrm{OH}$, interestingly peaking at the same place as $\mathrm{C}^{17} \mathrm{O}$ and showing a secondary peak toward the southwest part of the core where the emission of $\mathrm{C}^{17} \mathrm{O}$ also shows a decrease. This can be explained by the fact that, unlike $\mathrm{CH}_{3} \mathrm{OH}, \mathrm{H}_{2} \mathrm{CO}$ can also form via gas phase routes involving hydrocarbons (e.g., $\mathrm{CH}_{2}$ and $\mathrm{CH}_{3}$; Yamamoto 2017), which are expected to be abundant toward the southern part of the core, where the extinction abruptly drops to relatively low values and 
where carbon chains are in fact abundant (Spezzano et al. 2016; Ohashi et al. 1999).

The deuterated species of $\mathrm{H}_{2} \mathrm{CO}$ both peak at the center of the core, which is not the case for deuterated methanol, as seen in Fig. 3. Their distributions follow that of other deuterated species as $\mathrm{N}_{2} \mathrm{D}+$ and $\mathrm{NH}_{2} \mathrm{D}$ (Caselli et al. 2002b; Crapsi et al. 2007).

Interferometric observations are needed to make more detailed conclusions on possible differences in the distributions of the deuterated forms of methanol and formaldehyde; the resolution and sensitivity of these observations do not allow us to discuss them in locations at distances greater than where their emission is enhanced.

\subsection{Deuteration}

Previous results for the deuterium fractions measured in L1544 show a wide range of values: $\left[\mathrm{N}_{2} \mathrm{D}^{+}\right] /\left[\mathrm{N}_{2} \mathrm{H}^{+}\right]=0.2$, $\left[\mathrm{NH}_{2} \mathrm{D}\right] /\left[\mathrm{NH}_{3}\right]=0.5,\left[\mathrm{DCO}^{+}\right] /\left[\mathrm{HCO}^{+}\right]=0.04$, and $\left[\mathrm{c}-\mathrm{C}_{3} \mathrm{HD}\right] /$ $\left[\mathrm{c}-\mathrm{C}_{3} \mathrm{H}_{2}\right]=0.12-0.17$ (Caselli et al. 2002b; Crapsi et al. 2007; Spezzano et al. 2013). The $\mathrm{CH}_{2} \mathrm{DOH} / \mathrm{CH}_{3} \mathrm{OH}$ column density ratio never reaches values higher than 0.1 (with a peak value of $\sim 0.08 \pm 0.02)$, unlike $\left[\mathrm{N}_{2} \mathrm{D}^{+}\right] /\left[\mathrm{N}_{2} \mathrm{H}^{+}\right]$and $\left[\mathrm{NH}_{2} \mathrm{D}\right] /\left[\mathrm{NH}_{3}\right]$ (Crapsi et al. 2005, 2007). This suggests that deuterated methanol probably traces a more external layer than $\mathrm{N}_{2} \mathrm{D}^{+}$and $\mathrm{NH}_{3}$. This is analogous to the conclusion reached by Caselli et al. (2002b) to explain the $\left[\mathrm{DCO}^{+}\right] /\left[\mathrm{HCO}^{+}\right]$value since both $\mathrm{DCO}^{+}$and $\mathrm{HCO}^{+}$require gas-phase $\mathrm{CO}$ to form and $\mathrm{CO}$ is mainly frozen onto dust grains toward the core center, as already mentioned. The higher levels of deuteration measured in $\mathrm{N}_{2} \mathrm{H}^{+}$ and $\mathrm{NH}_{3}$ could also reflect the fact that these two molecules require more time to form compared to $\mathrm{CO}$ because of the slow formation rate of the parent molecule $\mathrm{N}_{2}$. Thus, molecular nitrogen becomes abundant only toward the central regions of the core where $\mathrm{CO}$ is mostly frozen and where $\mathrm{H}_{3}^{+}$is highly deuterated. As $\mathrm{CO}$ and related molecules (such as $\mathrm{CH}_{3} \mathrm{OH}$ and its deuterated forms) are mainly in the solid phase in these central regions (Caselli et al. 1999), deuterated methanol can only trace the zones surrounding the dust peak where the $\mathrm{D} / \mathrm{H}$ ratio is expected to be lower compared to the value deduced by the above-mentioned N-bearing species.

The deuterium fraction of methanol shows lower values than those found towards more evolved Class 0 objects. This difference with more evolved objects can be due to the presence of a major reservoir of $\mathrm{CH}_{2} \mathrm{DOH}$ in the ices in pre-stellar cores, which is released to the gas phase during the Class 0 phase (e.g., Parise et al. 2002, 2004). However, this scenario is still a matter of discussion due to recent results from high-resolution observations (e.g., Bianchi et al. 2017b; Persson et al. 2018), which show lower deuterium fractions for Class 0 objects.

Similarly, we find that $[\mathrm{HDCO}] /\left[\mathrm{H}_{2} \mathrm{CO}\right] \simeq 0.03 \pm 0.02$ and $\left[\mathrm{D}_{2} \mathrm{CO}\right] /\left[\mathrm{H}_{2} \mathrm{CO}\right] \simeq 0.04 \pm 0.03$ at the dust peak, which is consistent with previous values reported in pre-stellar cores and Class 0 objects (Bacmann et al. 2003; Parise et al. 2006). The value for $\left[\mathrm{D}_{2} \mathrm{CO}\right] /\left[\mathrm{H}_{2} \mathrm{CO}\right]$ is also consistent with that found by Bacmann et al. (2003). One thing to note is that, as in Bergman et al. (2011), we find a higher abundance of $\mathrm{D}_{2} \mathrm{CO}$ than HDCO. Bergman et al. (2011) claimed that this can only be explained if grain chemistry is ongoing, although their results are applied to the region of Ophiuchus, and the chemistry can differ from cloud to cloud. However, these molecules can suffer from depletion, which can be the source of discrepancy in the deuterium fractionations between different species, more importantly when compared with those that suffer less from depletion, like $\mathrm{N}_{2} \mathrm{D}^{+}$ and $\mathrm{NH}_{3}$.
As seen in Sect. 5, the model predictions for the deuteration of methanol in L1544 is higher than those observed, while the opposite happens with $\mathrm{D}_{2} \mathrm{CO}$. The deuteration of formaldehyde is close to that of methanol if the uncertainties are taken into account. Thus, more theoretical work needs to be done to fully understand deuteration processes in pre-stellar cores.

\section{Conclusions}

We have presented our maps of methanol, formaldehyde, and their deuterated species toward the well-known pre-stellar core L1544. These two molecules can help us to gain understanding of the chemical processes taking place on the dust grain surfaces and the formation of more complex organic molecules, and of the deuterium history in the process of star formation.

The highest level of deuteration of methanol occurs close to the dust peak, reaching $\left[\mathrm{CH}_{2} \mathrm{DOH}\right] /\left[\mathrm{CH}_{3} \mathrm{OH}\right]=0.8$. This indicates that a more external layer is traced by $\mathrm{CH}_{2} \mathrm{DOH}$ compared to $\mathrm{N}_{2} \mathrm{H}^{+}$(Caselli et al. 2002a) and $\mathrm{NH}_{3}$ (Crapsi et al. 2007). $\mathrm{CH}_{2} \mathrm{DOH}$ also shows a higher abundance at a distance of $\sim 3000$ au from the dust peak, exactly where more methanol is present in the gas phase with respect to $\mathrm{CO}$. This suggests that deuterated methanol is formed and released in the same way as $\mathrm{CH}_{3} \mathrm{OH}$.

$\mathrm{HDCO}$ and $\mathrm{D}_{2} \mathrm{CO}$, however, peak toward the center of the core, and present a large deuterium fraction, previously only found in $\rho$ Oph A (Bergman et al. 2011). Interestingly, $\mathrm{H}_{2} \mathrm{CO}$ shows a ring-like structure, depleting towards the center, and two maxima, one coinciding with $\mathrm{C}^{17} \mathrm{O}$ and a secondary peak toward the southwest; methanol, instead, coincides with a region where $\mathrm{C}^{17} \mathrm{O}$ shows less emission. This suggests that gas phase production via reactions involving hydrocarbons efficiently takes place in regions where $\mathrm{C}$ is not completely locked in $\mathrm{CO}$, based on the conclusions of Spezzano et al. (2016).

Finally, we compared two different chemical models with our observational results, and did not find an agreement. On the one hand, the model from Vasyunin et al. (2017) overproduces methanol and formaldehyde, probably caused by the overestimation of the efficiency of reactive desorption. On the other hand, the model from Sipilä et al. (2015a,b) does not produce enough methanol because of the slow diffusion of hydrogen atoms on the surfaces (which are not allowed to quantum tunnel) and it overproduces formaldehyde. Both models are static and the inclusion of dynamical evolution could change the results, although not by the orders of magnitude needed to reconcile models with observations. Our results suggest that quantum tunnelling for $\mathrm{H}$ diffusion on icy dust mantles should be considered, while reactive desorption still needs more detailed experimental work.

Higher sensitivity and angular resolution observations of the lines presented here are needed, together with a parameter-space exploration within current chemical models and laboratory work, to shed light on important chemical processes happening at the dawn of star formation.

Acknowledgements. The authors thank the anonymous referee for the useful comments, and the IRAM $30 \mathrm{~m}$ staff for their support in the observations. A.C.T. P.C., and J.E.P. acknowledge the financial support of the European Research Council (ERC; project PALs 320620). The work done by A.V. and A.P. was partially supported by the Russian Science Foundation (project 18-12-00351).

\section{References}

Aikawa, Y., Wakelam, V., Hersant, F., Garrod, R. T., \& Herbst, E. 2012, ApJ, 760,40

Astropy Collaboration (Robitaille, T. P., et al.) 2013, A\&A, 558, A33 
Bacmann, A., Lefloch, B., Ceccarelli, C., et al. 2003, ApJ, 585, L55

Bergin, E. A., \& Tafalla, M. 2007, ARA\&A, 45, 339

Bergman, P., Parise, B., Liseau, R., \& Larsson, B. 2011, A\&A, 527, A39

Bianchi, E., Codella, C., Ceccarelli, C., et al. 2017a, MNRAS, 467, 3011

Bianchi, E., Codella, C., Ceccarelli, C., et al. 2017b, A\&A, 606, L7

Bizzocchi, L., Caselli, P., Spezzano, S., \& Leonardo, E. 2014, A\&A, 569, A27

Bocquet, R., Demaison, J., Cosléou, J., et al. 1999, J. Mol. Spectrosc., 195, 345

Boogert, A. C. A., Gerakines, P. A., \& Whittet, D. C. B. 2015, ARA\&A, 53, 541

Caselli, P., \& Ceccarelli, C. 2012, A\&ARv., 20, 56

Caselli, P., Walmsley, C. M., Tafalla, M., Dore, L., \& Myers, P. C. 1999, ApJ, 523, L165

Caselli, P., Walmsley, C. M., Zucconi, A., et al. 2002a, ApJ, 565, 331

Caselli, P., Walmsley, C. M., Zucconi, A., et al. 2002b, ApJ, 565, 344

Caselli, P., van der Tak, F. F. S., Ceccarelli, C., \& Bacmann, A. 2003, A\&A, 403 L37

Ceccarelli, C., Caselli, P., Bockelée-Morvan, D., et al. 2014, Protostars and Planets VI (Tucson, AZ: University of Arizona Press), 859

Chacón-Tanarro, A., Caselli, P., Bizzocchi, L., et al. 2017, A\&A, 606, A142

Cornet, R., \& Winnewisser, G. 1980, J. Mol. Spectrosc., 80, 438

Crapsi, A., Caselli, P., Walmsley, C. M., et al. 2005, ApJ, 619, 379

Crapsi, A., Caselli, P., Walmsley, M. C., \& Tafalla, M. 2007, A\&A, 470, 221

Dalgarno, A., \& Lepp, S. 1984, ApJ, 287, L47

Emprechtinger, M., Caselli, P., Volgenau, N. H., Stutzki, J., \& Wiedner, M. C. 2009, A\&A, 493, 89

Keto, E., \& Caselli, P. 2010, MNRAS, 402, 1625

Keto, E., \& Rybicki, G. 2010, ApJ, 716, 1315

Keto, E., Rawlings, J., \& Caselli, P. 2014, MNRAS, 440, 2616

Keto, E., Caselli, P., \& Rawlings, J. 2015, MNRAS, 446, 3731

Klapper, G., Surin, L., Lewen, F., et al. 2003, ApJ, 582, 262

Linsky, J. L., Draine, B. T., Moos, H. W., et al. 2006, ApJ, 647, 1106

Mennella, V., Palumbo, M. E., \& Baratta, G. A. 2004, ApJ, 615, 1073

Mennella, V., Baratta, G. A., Palumbo, M. E., \& Bergin, E. A. 2006, ApJ, 643, 923

Minissale, M., Dulieu, F., Cazaux, S., \& Hocuk, S. 2016, A\&A, 585, A24

Müller, H. S., \& Lewen, F. 2017, J. Mol. Spectrosc., 331, 28

Muller, H. S. P., Gendriesch, R., Margules, L., et al. 2000, Phys. Chem. Chem. Phys., 2, 3401

Müller, H. S. P., Thorwirth, S., Roth, D. A., \& Winnewisser, G. 2001, A\&A, 370 , L49
Müller, H. S. P., Schlöder, F., Stutzki, J., \& Winnewisser, G. 2005, J. Mol. Struct., 742,215

Ohashi, N., Lee, S. W., Wilner, D. J., \& Hayashi, M. 1999, ApJ, 518, L41

Parise, B., Ceccarelli, C., Tielens, A. G. G. M., et al. 2002, A\&A, 393, L49

Parise, B., Castets, A., Herbst, E., et al. 2004, A\&A, 416, 159

Parise, B., Ceccarelli, C., Tielens, A. G. G. M., et al. 2006, A\&A, 453, 949

Parise, B., Leurini, S., Schilke, P., et al. 2009, A\&A, 508, 737

Pearson, J. C., Yu, S., \& Drouin, B. J. 2012, in 67th International Symposium on Molecular Spectroscopy, RF12

Persson, M. V., Jørgensen, J. K., Müller, H. S. P., et al. 2018, A\&A, 610, A54

Roberts, H., Herbst, E., \& Millar, T. J. 2003, ApJ, 591, L41

Ruffle, D. P., \& Herbst, E. 2001, MNRAS, 324, 1054

Sipilä, O., Caselli, P., \& Harju, J. 2015a, A\&A, 578, A55

Sipilä, O., Harju, J., Caselli, P., \& Schlemmer, S. 2015b, A\&A, 581, A122

Sipilä, O., Spezzano, S., \& Caselli, P. 2016, A\&A, 591, L1

Spezzano, S., Brünken, S., Schilke, P., et al. 2013, ApJ, 769, L19

Spezzano, S., Bizzocchi, L., Caselli, P., Harju, J., \& Brünken, S. 2016, A\&A, 592, L11

Tafalla, M., Mardones, D., Myers, P. C., et al. 1998, ApJ, 504, 900

Tafalla, M., Myers, P. C., Caselli, P., Walmsley, C. M., \& Comito, C. 2002, ApJ, 569,815

Taquet, V., Ceccarelli, C., \& Kahane, C. 2012, ApJ, 748, L3

Tielens, A. G. G. M., \& Hagen, W. 1982, A\&A, 114, 245

van der Walt, S., Colbert, S. C., \& Varoquaux, G. 2011, Comput. Sci. Eng., 13, 22

Vastel, C., Ceccarelli, C., Lefloch, B., \& Bachiller, R. 2014, ApJ, 795, L2

Vasyunin, A. I., \& Herbst, E. 2013, ApJ, 769, 34

Vasyunin, A. I., Sobolev, A. M., Wiebe, D. S., \& Semenov, D. A. 2004, Astron. Lett., 30, 566

Vasyunin, A. I., Semenov, D., Henning, T., et al. 2008, ApJ, 672, 629

Vasyunin, A. I., Caselli, P., Dulieu, F., \& Jiménez-Serra, I. 2017, ApJ, 842, 33

Walmsley, C. M., Flower, D. R., \& Pineau des Forêts, G. 2004, A\&A, 418, 1035

Watanabe, N., \& Kouchi, A. 2002, ApJ, 571, L173

Whittet, D. C. B., Gerakines, P. A., Tielens, A. G. G. M., et al. 1998, ApJ, 498, L159

Willacy, K., Langer, W. D., \& Velusamy, T. 1998, ApJ, 507, L171

Wilson, T. L., \& Rood, R. 1994, ARA\&A, 32, 191

Xu, L.-H., \& Lovas, F. J. 1997, J. Phys. Chem. Ref. Data, 26, 17

Yamamoto, S. 2017, Introduction to Astrochemistry: Chemical Evolution from Interstellar Clouds to Star and Planet Formation (Tokyo: Springer) 


\section{Appendix A: Integrated intensity maps}
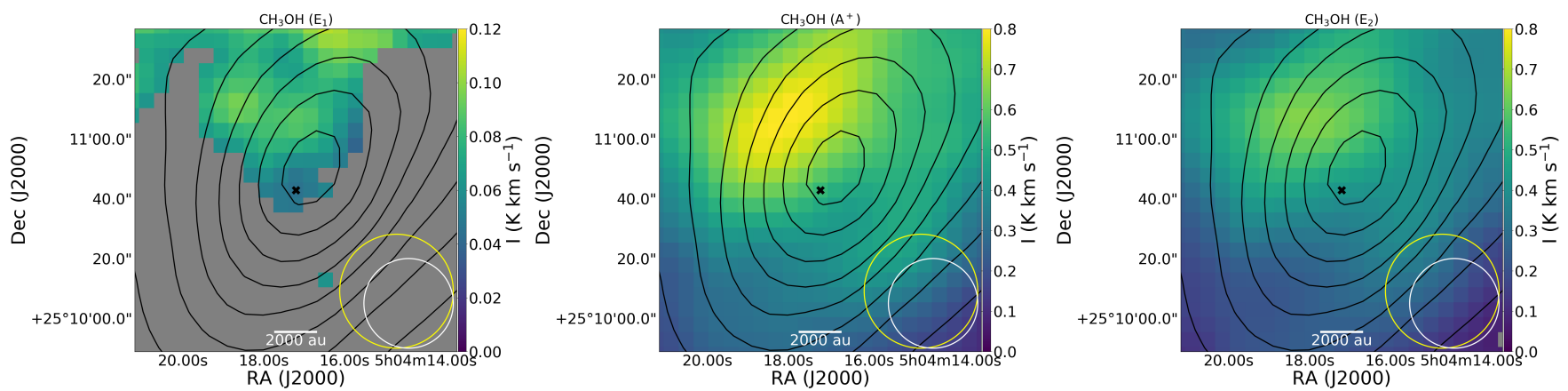

Fig. A.1. Integrated intensity maps of the three methanol transitions observed: $\mathrm{E}_{1}$ (left panel), $\mathrm{A}^{+}$(middle panel), and $\mathrm{E}_{2}$ (right panel). Only pixels with flux values above a $3 \sigma$ detection level are presented. The black contours represent increasing $10 \%$ steps of the $\mathrm{N}_{\mathrm{H}_{2}}$ column density map, derived by Spezzano et al. (2016). The noise in the integrated intensities is $0.02 \mathrm{~K} \mathrm{~km} \mathrm{~s}^{-1}$. The beams are shown in the bottom right corner of the figures, in yellow for Herschel/SPIRE and in white for the $30 \mathrm{~m}$ telescope. The black cross marks the dust continuum peak.

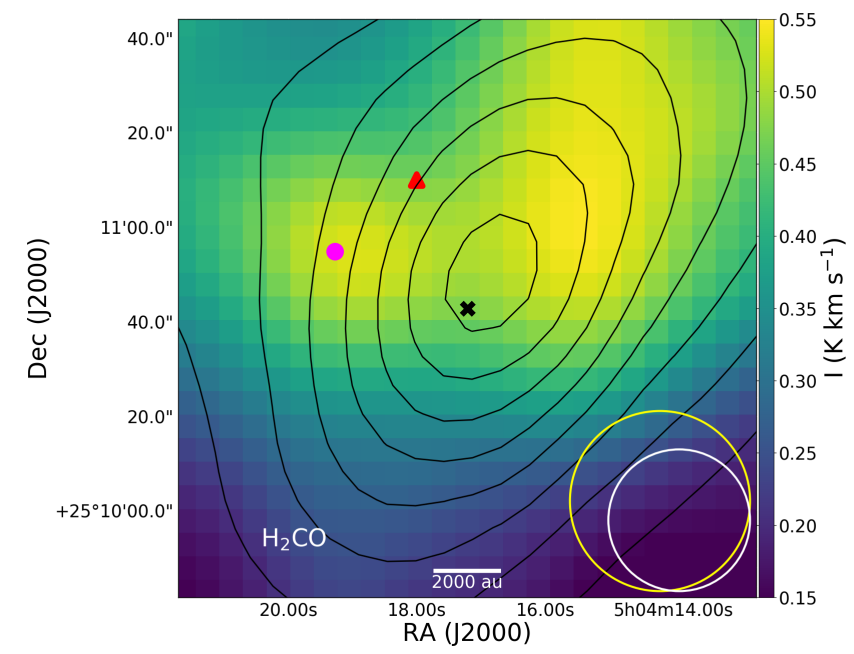

Fig. A.2. Integrated intensity map of the $\mathrm{H}_{2} \mathrm{CO}\left(2_{1,2}-1_{1,1}\right)$ line. The black contours represent increasing $10 \%$ steps of the $\mathrm{N}_{\mathrm{H}_{2}}$ column density map, derived by Spezzano et al. (2016). The error on the integrated intensity is $0.014 \mathrm{~K} \mathrm{~km} \mathrm{~s}^{-1}$. The beams are shown in the bottom right corner of the figure, in yellow for Herschel/SPIRE and in white for the $30 \mathrm{~m}$ telescope. The black cross marks the dust continuum peak, the red triangle the methanol peak, and the pink circle the $\mathrm{C}^{17} \mathrm{O}$ peak. 


\section{Appendix B: Column density maps}

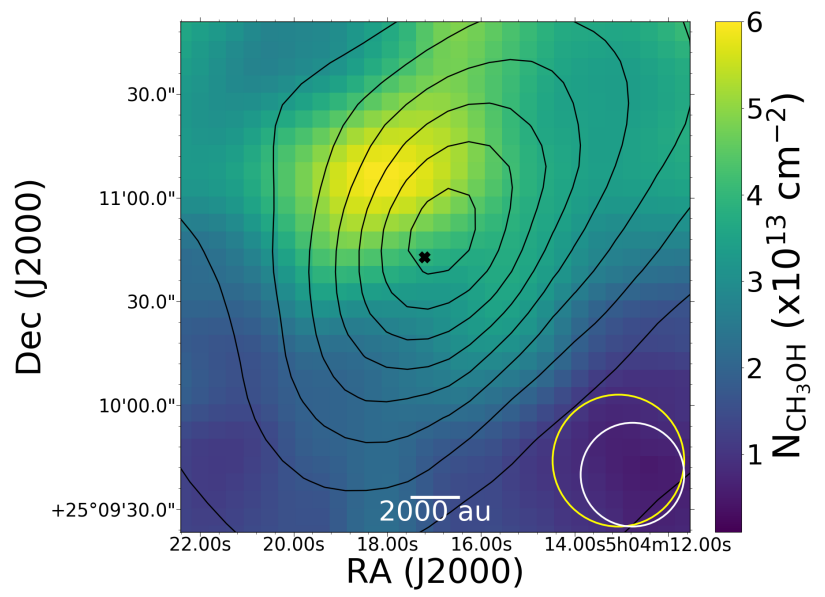

Fig. B.1. Column density map of $\mathrm{CH}_{3} \mathrm{OH}$ derived as explained in Sect. 4.1. The black contours represent increasing 10\% steps of the $\mathrm{N}_{\mathrm{H}_{2}}$ column density map, derived by Spezzano et al. (2016). The beams are shown in the bottom right corner of the figure, in yellow for Herschel/SPIRE and in white for the $30 \mathrm{~m}$ telescope. The black cross marks the dust continuum peak.

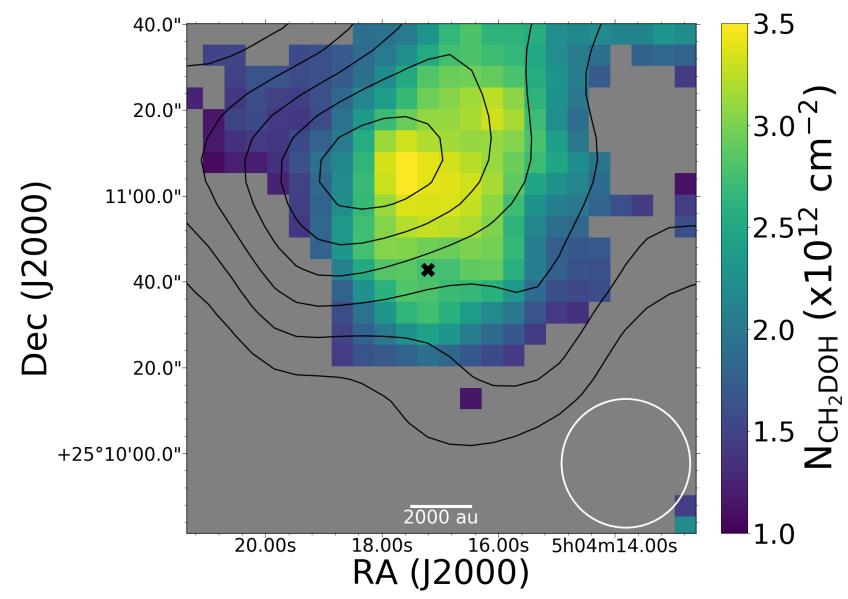

Fig. B.2. Column density map of $\mathrm{CH}_{2} \mathrm{DOH}$ derived as explained in Sect. 4.1. The black contours represent $10 \%$ steps in the $\mathrm{CH}_{3} \mathrm{OH}$ column density. The black cross marks the dust continuum peak. The beam is shown in the bottom right corner.

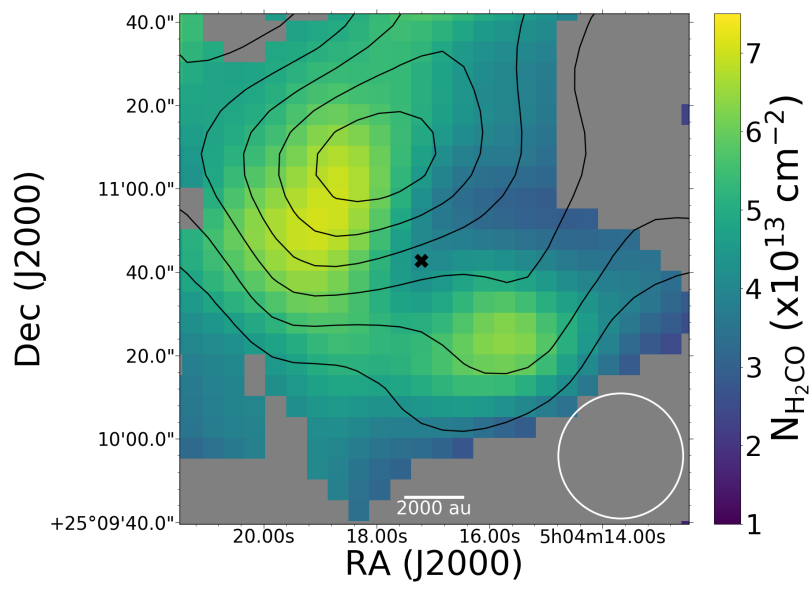

Fig. B.3. Column density map of $\mathrm{H}_{2} \mathrm{CO}$ derived as explained in Sect. 4.1. The black contours represent $10 \%$ steps in the $\mathrm{CH}_{3} \mathrm{OH}$ column density. The black cross marks the dust continuum peak. The beam is shown in the bottom right corner.

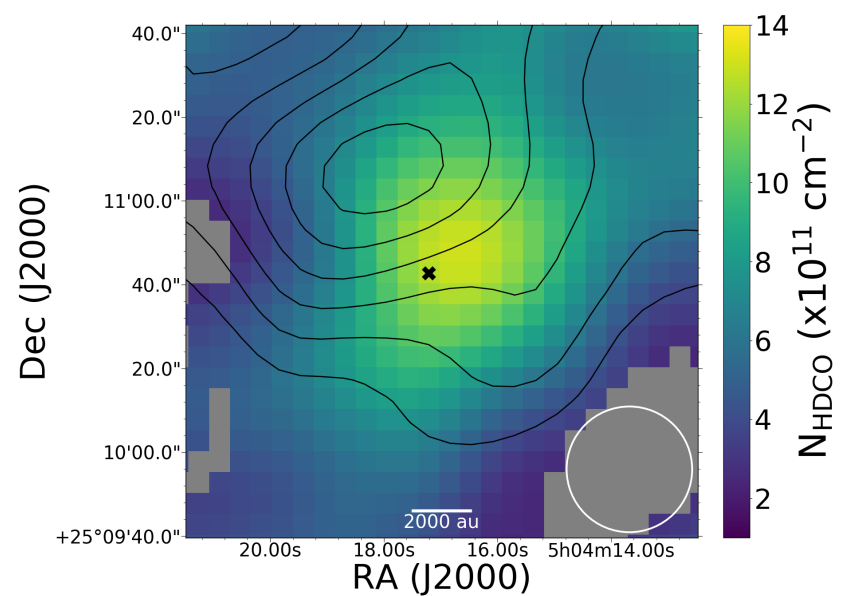

Fig. B.4. Column density map of HDCO derived as explained in Sect. 4.1. The black contours represent $10 \%$ steps in the $\mathrm{CH}_{3} \mathrm{OH}$ column density. The black cross marks the dust continuum peak. The beam is shown in the bottom right corner.

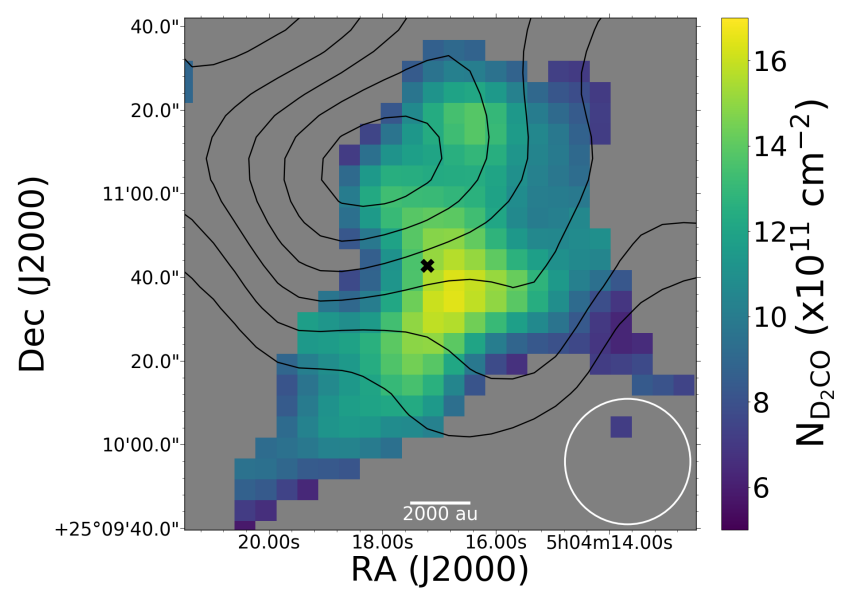

Fig. B.5. Column density map of $\mathrm{D}_{2} \mathrm{CO}$ derived as explained in Sect. 4.1. The black contours represent $10 \%$ steps in the $\mathrm{CH}_{3} \mathrm{OH}$ column density. The black cross marks the dust continuum peak. The beam is shown in the bottom right corner.

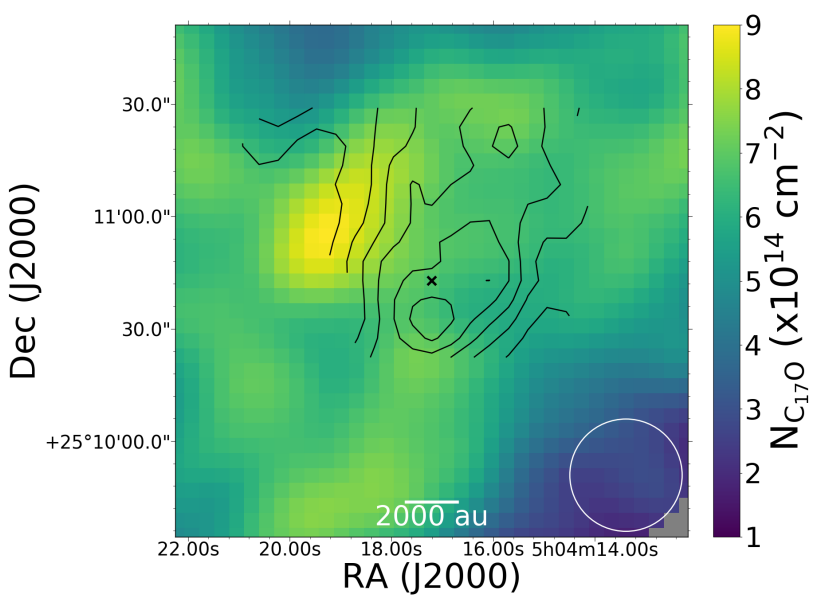

Fig. B.6. Column density map of $\mathrm{C}^{17} \mathrm{O}$ derived as explained in Sect. 4.1. The black contours represent $10 \%$ steps in the methanol deuterium fraction. The black cross marks the dust continuum peak. The beam is shown in the bottom right corner. 
A. Chacón-Tanarro et al.: Mapping deuterated methanol toward L1544. I. Deuterium fraction and comparison with modeling

\section{Appendix C: Errors on the deuteration maps}

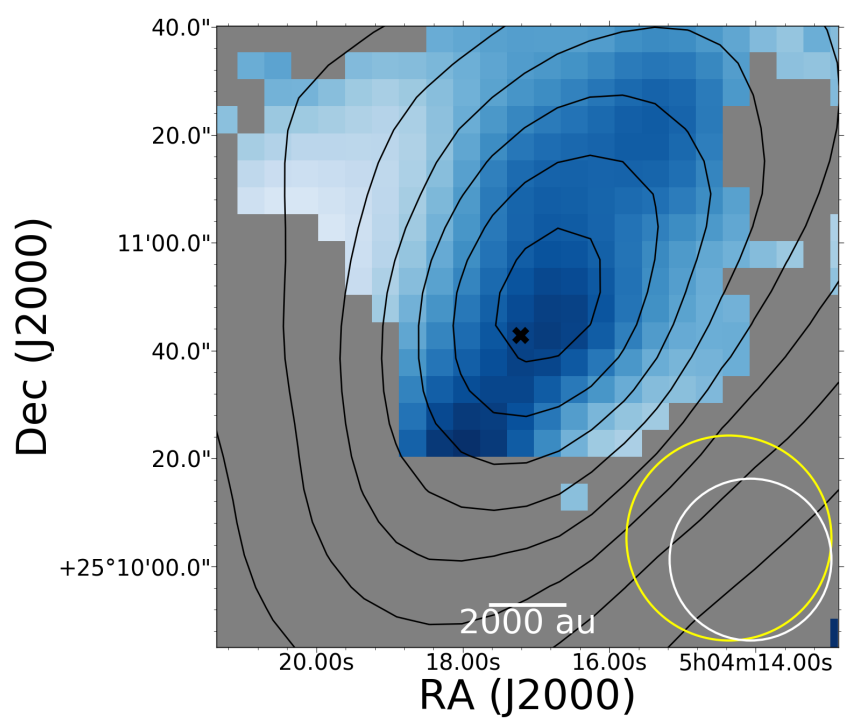

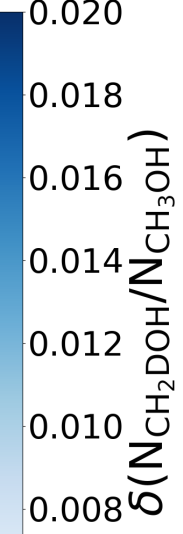

0.006
Fig. C.1. Error on the ratio between $\mathrm{N}\left(\mathrm{CH}_{2} \mathrm{DOH}\right)$ and $\mathrm{N}\left(\mathrm{CH}_{3} \mathrm{OH}\right)$. The black contours represent increasing $10 \%$ steps of the peak of the Herschel $\mathrm{N}\left(\mathrm{H}_{2}\right)$ map, presented by Spezzano et al. (2016). The beams are shown in the bottom right corner of the figure, in yellow for Herschel/SPIRE and in white for the $30 \mathrm{~m}$ telescope. The black cross marks the dust continuum peak.
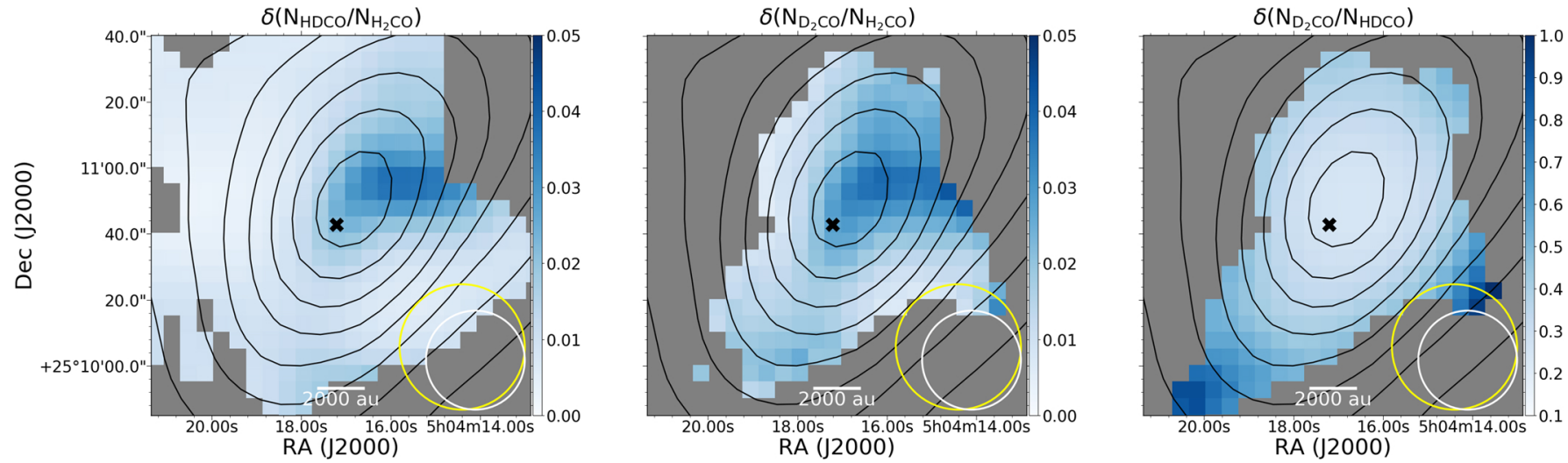

Fig. C.2. Error on the ratio between $\mathrm{N}(\mathrm{HDCO})$ and $\mathrm{N}\left(\mathrm{H}_{2} \mathrm{CO}\right.$ ) (left panel), $\mathrm{N}\left(\mathrm{D}_{2} \mathrm{CO}\right)$ and $\mathrm{N}\left(\mathrm{H}_{2} \mathrm{CO}\right)$ (middle panel), and $\mathrm{N}\left(\mathrm{D}_{2} \mathrm{CO}\right)$ and $\mathrm{N}(\mathrm{HDCO})$ (right panel). The black contours represent increasing $10 \%$ steps of the peak of the Herschel $\left.\mathrm{N}_{(} \mathrm{H}_{2}\right)$ map, presented by Spezzano et al. (2016). The beams are shown in the bottom right corner of the figures, in yellow for Herschel/SPIRE and in white for the $30 \mathrm{~m}$ telescope. The black cross marks the dust continuum peak. 